\title{
ON THE PROJECTION OF FUNCTIONS INVARIANT UNDER THE ACTION OF A CRYSTALLOGRAPHIC GROUP
}

\author{
ELIANA M. PINHO, ISABEL S. LABOURIAU
}

\begin{abstract}
We study functions defined in $(n+1)$-dimensional domains that are invariant under the action of a crystallographic group. We give a complete description of the symmetries that remain after projection into an $n$ dimensional subspace and compare it to similar results for the restriction to a subspace. We use the Fourier expansion of invariant functions and the action of the crystallographic group on the space of Fourier coefficients. Intermediate results relate symmetry groups to the dual of the lattice of periods.
\end{abstract}

Addresses:

\author{
Eliana M. Pinhoa1 Isabel S. Labouriau ${ }^{\text {a }}$ \\ ${ }^{a}$ Centro de Matemática and \\ Departamento de Matemática, Faculdade de Ciências \\ Universidade do Porto \\ Rua do Campo Alegre 687, 4169-007 Porto, Portugal.
}

E. M. Pinho email empinho@gmail.com

I.S.Labouriau email islabour@fc.up.pt — Corresponding author — telephone $(+351) 226063314$

2010 Mathematics Subject Classification. Primary 20H15 ; Secondary 52C22, 58D19, 37G40. Key words and phrases. Crystallographic groups; lattices; tilings in $n$ dimensions; Patterns and functions: symmetric, periodic, projected, restricted; Fourier coefficients.

${ }^{1}$ Present address: CEAU Centro de Estudos em Arquitectura e Urbanismo da Faculdade de Arquitectura da Universidade do Porto, Rua do Gólgota, 215, 4150-755 Porto, Portugal. 


\section{INTRODUCTION}

We study real functions with domain $\mathbf{R}^{n+1}$ that are invariant under the action of a crystallographic group $\Gamma$, a group with a subgroup of translations that is a lattice $\mathcal{L}$ containing $n+1$ linearly independent elements.

A crystallographic group $\Gamma$ is a special type of subgroup of the Euclidean group $\mathbf{E}(n+1)$, the semi-direct product $\mathbf{E}(n+1) \cong \mathbf{R}^{n+1} \ltimes \mathbf{O}(n+1)$. We denote its elements $\gamma=(v, \delta)$, where $v \in \mathbf{R}^{n+1}$ and $\delta \in \mathbf{O}(n+1)$.

Let $\Gamma$ be a crystallographic group and let $X_{\Gamma}$ be the space of all $\Gamma$-invariant functions of class $C^{1}$ on $\mathbf{R}^{n+1}$, that in particular are $\mathcal{L}$-periodic. Using coordinates $(x, y)$ in $\mathbf{R}^{n+1}$ with $x \in \mathbf{R}^{n}, y \in \mathbf{R}$ and for $y_{0}>0$, the projection operator $\Pi_{y_{0}}$ integrates a function $f$ along the width $y_{0}$, yielding a new function with domain $\mathbf{R}^{n}$ :

$$
\Pi_{y_{0}}(f)(x)=\int_{0}^{y_{0}} f(x, y) d y .
$$

Our main result, Theorem 1.1, relates the symmetry of the functions $f \in X_{\Gamma}$ to the the group $\Sigma_{y_{0}}$ of symmetries of the projected functions $\Pi_{y_{0}}\left(X_{\Gamma}\right)$, i.e., the largest subgroup of $\mathbf{E}(n) \cong \mathbf{R}^{n} \ltimes \mathbf{O}(n)$ that fixes all the elements in $\Pi_{y_{0}}\left(X_{\Gamma}\right)$.

The elements of $\mathbf{O}(n+1)$ that map $\mathbf{R}^{n} \times\{0\}$ into itself are, for $\alpha \in \mathbf{O}(n)$ :

$$
\sigma=\left(\begin{array}{cc}
I d_{n} & 0 \\
0 & -1
\end{array}\right), \quad \alpha_{+}=\left(\begin{array}{cc}
\alpha & 0 \\
0 & 1
\end{array}\right) \quad \text { and } \quad \alpha_{-}=\sigma \alpha_{+}=\left(\begin{array}{cc}
\alpha & 0 \\
0 & -1
\end{array}\right) .
$$

Consider the subgroup $\widehat{\Gamma}$ of elements of $\Gamma$ with orthogonal part $\alpha_{ \pm}$for some $\alpha \in$ $\mathbf{O}(n)$ of the form

$$
\left((v, y),\left(\begin{array}{cc}
\alpha & 0 \\
0 & \beta
\end{array}\right)\right) \quad \text { with } \quad \beta= \pm 1 \quad v \in \mathbf{R}^{n} \quad y \in \mathbf{R} .
$$

In particular, $\widehat{\Gamma}$ contains all the vertical translations $\left((0, y), I d_{n+1}\right)$ and vertical reflections $((0, y), \sigma)$ of $\Gamma$. Let $h: \widehat{\Gamma} \longrightarrow \mathbf{E}(n) \cong \mathbf{R}^{n} \ltimes \mathbf{O}(n)$ be the projection

$$
h\left((v, y),\left(\begin{array}{cc}
\alpha & 0 \\
0 & \beta
\end{array}\right)\right)=(v, \alpha) .
$$

Theorem 1.1 states that the elements in $\Gamma$ that effectively contribute to the symmetry of $\Pi_{y_{0}}\left(X_{\Gamma}\right)$ are those in the subgroup $\Gamma_{y_{0}}$ of $\widehat{\Gamma}$ with the following description:

- If $\left(0, y_{0}\right) \in \mathcal{L}$ then $\Gamma_{y_{0}}=\widehat{\Gamma}$.

- If $\left(0, y_{0}\right) \notin \mathcal{L}$ then $\Gamma_{y_{0}}$ contains those elements of $\widehat{\Gamma}$ that are either of the form $\left((v, 0), \alpha_{+}\right)$or of the form $\left(\left(v, y_{0}\right), \alpha_{-}\right)$.

Theorem 1.1. The group $\Sigma_{y_{0}}$ of symmetries of the projected functions $\Pi_{y_{0}}\left(X_{\Gamma}\right)$ is the image of $\Gamma_{y_{0}}$ by the projection $h: \widehat{\Gamma} \longrightarrow \mathbf{E}(n)$, a group homomorphism whose kernel is given by the vertical translations and vertical reflections lying in $\Gamma_{y_{0}}$.

Theorem 1.1 is obtained as a corollary to the following criterion that describes how elements of $\Gamma_{y_{0}}$ are transformed by the projection:

Theorem 1.2. All functions in $\Pi_{y_{0}}\left(X_{\Gamma}\right)$ are invariant under the action of $\left(v_{\alpha}, \alpha\right) \in$ $\mathbf{R}^{n} \ltimes \mathbf{O}(n)$ if and only if one of the following conditions holds:

(I) $\left(\left(v_{\alpha}, 0\right), \alpha_{+}\right) \in \Gamma$

(II) $\left(\left(v_{\alpha}, y_{0}\right), \alpha_{-}\right) \in \Gamma$,

(III) $\left(0, y_{0}\right) \in \mathcal{L}$, and either $\left(\left(v_{\alpha}, y_{1}\right), \alpha_{+}\right) \in \Gamma$ or $\left(\left(v_{\alpha}, y_{1}\right), \alpha_{-}\right) \in \Gamma$, for some $y_{1} \in \mathbf{R}$.

For the proof of Theorem 1.2 we work with the induced action of $\Gamma$ on the Fourier expansion of functions in $X_{\Gamma}$ in terms of the waves $\omega_{k}(x, y)=\mathrm{e}^{2 \pi i<k,(x, y)>}$, with $x \in \mathbf{R}^{n}$ and $y \in \mathbf{R}, k \in \mathbf{R}^{n+1}$. The result only depends on the formal expansion, 
and also holds under less restrictive hypotheses on $X_{\Gamma}$, given in Section 2 below. The particular case of a projection from the plane into a line has been treated in [12], where a different proof of Theorem 1.2 is given for the case $n=1$. In this dimension, the only symmetries that remain after projection are translations and reflections, which simplifies the analysis. The results in the present article may also be found in the thesis of Pinho [16], sometimes in a different form.

We are interested in comparing the symmetries of projected functions to those of their restriction to a hyperplane. For $r \in \mathbf{R}$, let $\Phi_{r}$ be the operator that maps $f(x, y)$ to its restriction to the affine subspace $\left\{(x, r): x \in \mathbf{R}^{n}\right\}$, given by $\Phi_{r}(f)(x)=f(x, r)$. Let $G_{r}$ be the largest subgroup of $\mathbf{E}(n) \cong \mathbf{R}^{n} \ltimes \mathbf{O}(n)$ that fixes all the elements in $\Phi_{r}\left(X_{\Gamma}\right)$, and let $\widetilde{\Gamma}_{2 r}$ be the subgroup of $\widehat{\Gamma}$ of elements that are either of the form $\left((v, 0), \alpha_{+}\right)$or of the form $\left((v, 2 r), \alpha_{-}\right)$.

Theorem 1.3. The group $G_{r}$ of symmetries of the restriction $\Phi_{r}\left(X_{\Gamma}\right)$ of functions in $X_{\Gamma}$ to a hyperplane is the image of $\widetilde{\Gamma}_{2 r}$ by the group homomorphism $h: \widehat{\Gamma} \longrightarrow$ $\mathbf{E}(n)$ of Theorem 1.1.

1.1. Motivation. The main idea of this article is to track the symmetries of functions when they undergo transformations, as projections and restrictions. Some of the symmetries may get mixed in the transformed functions, others may disappear, sometimes leaving hidden traces.

In experiments, the characteristics of objects may not allow direct observation of their properties, but enable access to their projections or restrictions. In this situation there is a loss of data, and the observed properties are to be interpreted as traces of the actual features of higher dimension solutions. Our results clarify this interpretation in what concerns symmetry.

The results in this article were motivated by the study of patterns in reactiondiffusion experiments on thin layers, where the observation method carries information from the depth of a layer and thus corresponds to a projection whose role in the formation of a pattern is not always clear (see Gomes [10], De Kepper et al. [6], Borckmans et al. [2] and other articles by the same authors). Our results are a first step in an attempt to clarify this issue.

Models for patterns in thin three-dimensional layers often make the simplifying assumption that the domain is two-dimensional (see, for instance Gunaratne et al. [11]). In many cases the simplified model gives a good reproduction of experimental results, as in those discussed by Golubitsky and Stewart [9, Chapter 5]. There are situations, however, when not all experimentally observed patterns may be obtained from this analysis, as indicated by Gomes [10] who proposes that some of these patterns may arise as the projection of a three-dimensional periodic structure. Knowledge of projected patterns is useful when deciding whether this is the case, as in Zhou et al. [20]. When the thickness of the layer acts as a bifurcation parameter, as observed by De Kepper et al. [6], then the symmetries of the pattern may be subject to change as thickness varies. This may be of particular importance in the study of patterns in growing domains.

In this context, the standard method for analysing patterns is to describe them as solutions of partial differential equations. The general problem is too complicated to solve (for a discussion, see Dione and Golubitsky [7]) so one looks for solutions that are periodic in the spatial variables with periods in a lattice $\mathcal{L}$. Under general assumptions, this reduces the problem to an ordinary differential equation in a finite-dimensional space. The $\mathcal{L}$-periodic solutions of partial differential equations arise in many problems, either as solutions on their own right or by the decomposition of other solutions. 
The explicit reduction of the problem to a finite-dimensional space may be difficult to compute. Instead, one uses the general form of ordinary differential equations with the prescribed symmetry. This general form may then be analysed to decide whether there is, generically, a bifurcation to a certain pattern. Knowledge of the symmetries is thus an essential step in dealing with this problem. For a more detailed treatment we refer the reader to [9, Chapter 5] and [11] where the 2-dimensional case is discussed, to [7] and, for issues arising from a projection, to $[10]$.

When dealing with the problem of deciding whether a pattern in a thin domain may be explained by a 2-dimensional model, it is possible, in theory, to carry out the analysis in 3 spatial dimensions, project the results to 2 spatial dimensions and then compare it to generic models formulated directly in the lower-dimensional space. However, it is a lot easier to compare systems formulated in the same dimension, having different symmetries. Moreover, problems formulated in higher dimension are more difficult. The purpose of this article is to provide the tools for performing this type of analysis directly in the lower dimension. Theorem 1.1 ensures that none of the symmetries are left out.

Symmetries that do not remain after projection may give rise to properties of the projected functions that cannot be described as the invariance under a crystallographic group. An illustrative example is the quasiperiodic structure obtained by the canonical projection of a periodic one (see example 2 of section 9 at the end of this article and, more generally, Senechal [17, section 2.6]). The canonical projection ensures a quasiperiodical result and stands on strict conditions: one has to start from an integral lattice and project into a hyperplane that only meets the lattice at the origin. The subset of the lattice that is projected is also fixed. For an integral lattice, if the restriction $\Phi_{r}$ with $r=0$ is only the origin, then it is not possible to have a codimension one periodic projection. However, we show that this may happen for more general lattices if $\left(0, y_{0}\right) \in \mathcal{L}$ as in example 4 of section 9 . Our results may frame the canonical projection, and thus the quasiperiodical structures, through the variation of parameters on the definition of the lattice and also of the projection.

1.2. Structure of the article. Section 2 contains definitions, notation and some preliminary results. In particular, we formulate minimal assumptions on the space $X_{\Gamma}$ for Theorems 1.1 and 1.2. The formulation of the results for sufficiently large spaces of $\Gamma$-invariant functions highlights their common characteristic, the symmetry.

Each one of the conditions (I), (II) and (III) of Theorem 1.2 is sufficient by basic properties of integrals, as shown in section 3 .

The bulk of the paper contains the proof that the conditions of Theorem 1.2 are necessary. As usual, it is harder to prove that a list is complete than to show that some elements are on a list. We state first, in section 4 , a formulation of the symmetries in $\Sigma_{y_{0}}$ in terms of restrictions on $\Gamma$ and on the dual lattice $\mathcal{L}^{*}$, Proposition 4.1. Then, after establishing some technical results in section 5 , we show in section 6 that these properties impose restrictions on $\Gamma$ and on $\mathcal{L}$ by implying the presence of some particular elements in $\Gamma$, establishing Theorem 1.2 as a consequence of Proposition 4.1. The second result is proved in section 7 using the induced action of $\Gamma$ in the space of Fourier coefficients of $\Gamma$-invariant functions, that appears as relations on the coefficients that may be traced after projection.

Finally, in section 8 a similar study is carried out for the symmetries of the restriction of invariant functions to a hyperplane. This is useful in comparing the restriction and projection of a pattern. The main result of this section is 
Theorem 1.3, and this follows from Theorem 8.1, an analogue to Theorem 1.2, that is proved using simpler versions of the arguments developed for the projection.

Examples of the effect of projection and restriction for some crystallographic groups are given in section 9 .

\section{Notation and Preliminary Results}

The reader is referred to Armstrong [1] for results on Euclidean and plane crystallographic groups, to Senechal [17] and Miller [14] for results on lattices and crystallographic groups. A detailed description may be found in Pinho [16].

The action of an element $(v, \delta)$ of the Euclidean group $\mathbf{E}(n+1)$ on $(x, y), x \in \mathbf{R}^{n}$, $y \in \mathbf{R}$ is given by $(v, \delta) \cdot(x, y)=v+\delta(x, y)$ with the group operation $\left(v_{1}, \delta_{1}\right)$. $\left(v_{2}, \delta_{2}\right)=\left(v_{1}+\delta_{1} v_{2}, \delta_{1} \delta_{2}\right)$, for $\left(v_{1}, \delta_{1}\right),\left(v_{2}, \delta_{2}\right) \in \mathbf{E}(n+1)$.

A crystallographic group $\Gamma$ with lattice $\mathcal{L}$ is a subgroup $\Gamma \leq \mathbf{E}(n+1)$ such that the orbit of the origin by translations $\left\{v:\left(v, I d_{n+1}\right) \in \Gamma\right\}$ is a $\mathbf{Z}$-module generated by $n+1$ linearly independent vectors $l_{1}, \ldots, l_{n+1} \in \mathbf{R}^{n+1}: \mathcal{L}=\left\{l_{1}, \ldots, l_{n+1}\right\}_{\mathbf{z}}=$ $\left\{\sum_{i=1}^{n+1} m_{i} l_{i}: m_{i} \in \mathbf{Z}\right\}$. We also use the symbol $\mathcal{L}$ for the normal subgroup of translations of $\Gamma$ isomorphic to $(\mathcal{L},+)$.

The projection $(v, \delta) \longmapsto \delta$, of $\Gamma$ into $\mathbf{O}(n+1)$, has kernel $\mathcal{L}$. Its image, $\mathbf{J}=$ $\left\{\delta:(v, \delta) \in \Gamma\right.$ for some $\left.v \in \mathbf{R}^{n+1}\right\}$, called the point group of $\Gamma$, is isomorphic to the finite quotient $\Gamma / \mathcal{L}$ and is a subgroup of the holohedry of $\mathcal{L}$, the largest subgroup of $\mathbf{O}(n+1)$ that leaves $\mathcal{L}$ invariant. Thus, $\mathbf{J} \mathcal{L}=\{\delta l: \delta \in \mathbf{J}, l \in \mathcal{L}\}=\mathcal{L}$.

The set of all the elements in $\Gamma$ with orthogonal component $\delta \in \mathbf{J}$ is the coset $\mathcal{L} \cdot(v, \delta)=\{(l+v, \delta): l \in \mathcal{L}\}$ for any $v \in \mathbf{R}^{n+1}$ such that $(v, \delta) \in \Gamma$. The group $\Gamma$ is characterized by the $n+1$ generators of $\mathcal{L}$, plus a finite number of elements $\left(v_{\delta}, \delta\right)$, with $\delta \in \mathbf{J}$.

The action of $\Gamma$ in $\mathbf{R}^{n+1}$ induces the scalar action on functions: $(\gamma \cdot f)(x, y)=$ $f\left(\gamma^{-1} \cdot(x, y)\right)$ for $\gamma \in \Gamma$ and $(x, y) \in \mathbf{R}^{n+1}$, see Melbourne [13]. A function $f$ is $\Gamma$-invariant if $(\gamma \cdot f)(x, y)=f(x, y)$, for all $\gamma \in \Gamma$ and all $(x, y) \in \mathbf{R}^{n+1}$.

The dual lattice of $\mathcal{L}$ is the set of all the elements $k \in \mathbf{R}^{n+1}$ such that the waves $\omega_{k}(x, y)=\mathrm{e}^{2 \pi i<k,(x, y)>}$ are $\mathcal{L}$-periodic. It is given by $\mathcal{L}^{*}=\left\{k \in \mathbf{R}^{n+1}:<k, l_{i}>\epsilon\right.$ $\mathbf{Z}, i=1, \ldots, n+1\}$, where $\langle\cdot, \cdot\rangle$ denotes the usual inner product in $\mathbf{R}^{n+1}$. It may be written as $\mathcal{L}^{*}=\left\{l_{1}^{*}, \ldots, l_{n+1}^{*}\right\} \mathbf{z}$, where the dual basis $l_{i}^{*} \in \mathbf{R}^{n+1}$ satisfies $<l_{i}^{*}, l_{j}>=\delta_{i j}$ for all $i, j \in\{1, \ldots, n+1\}$. This representation as inner product depends on the choice of basis in $\mathcal{L}$. The lattices $\mathcal{L}$ and $\mathcal{L}^{*}$ have the same holohedry.

For our purposes, the relevant property of functions $f \in X_{\Gamma}$ is the existence of a Fourier expansion in terms of the waves $\omega_{k}(x, y)$

$$
f(x, y)=\sum_{k \in \mathcal{L}^{*}} \omega_{k}(x, y) C(k)
$$

where $C: \mathcal{L}^{*} \longrightarrow \mathbf{C}$ are the Fourier coefficients. For a real function $f$ we have $\overline{C(k)}=C(-k)$. From the action of $\Gamma$ on $X_{\Gamma}$ we get:

$$
\begin{aligned}
(v, \delta) \cdot f(x, y) & =\sum_{k \in \mathcal{L}^{*}} \omega_{\delta k}(x, y) \omega_{\delta k}(-v) C(k), \text { by orthogonality of } \delta, \\
& =\sum_{k \in \mathcal{L}^{*}} \omega_{k}(x, y) \omega_{k}(-v) C\left(\delta^{-1} k\right), \text { because } \delta \mathcal{L}^{*}=\mathcal{L}^{*} .
\end{aligned}
$$

This induces, on the space of Fourier coefficients, an action of $\Gamma$ with centraliser $\mathcal{L}$, given by $(v, \delta) \cdot C(k)=\omega_{k}(-v) C\left(\delta^{-1} k\right)$. The $(v, \delta)$-invariance of $f$ implies $C(k)=\omega_{k}(-v) C\left(\delta^{-1} k\right)$ for all its Fourier coefficients.

The simplest $\Gamma$-invariant functions are the real and imaginary components of $I_{k}(x, y)=\sum_{\delta \in \mathbf{J}} \omega_{\delta k}(x, y) \omega_{\delta k}\left(-v_{\delta}\right)$, for $k \in \mathcal{L}^{*},\left(v_{\delta}, \delta\right) \in \Gamma$, and we will assume that they lie in $X_{\Gamma}$. Each function $I_{k}$, for $k \in \mathcal{L}^{*}$, is the sum of the elements in the J-orbit of $\omega_{k}$. Note that the expression of $I_{k}$ does not depend on the choice of $v_{\delta}$. 
For $f \in X_{\Gamma}$ we get $\Pi_{y_{0}}(f)(x)=\int_{0}^{y_{0}} \sum_{k \in \mathcal{L}^{*}} \omega_{k}(x, y) C(k) d y$. When the integral and the summation commute, then

$$
\begin{aligned}
\Pi_{y_{0}}(f)(x) & =\sum_{k \in \mathcal{L}^{*}} \int_{0}^{y_{0}} \omega_{k}(x, y) C(k) d y \\
& =\sum_{k \in \mathcal{L}^{*}} \omega_{k_{1}}(x) C\left(k_{1}, k_{2}\right) \int_{0}^{y_{0}} \omega_{k_{2}}(y) d y
\end{aligned}
$$

where $k=\left(k_{1}, k_{2}\right)$, with $k_{1} \in \mathbf{R}^{n}$ and $k_{2} \in \mathbf{R}$. Grouping terms with common $n$ first components in $\mathcal{L}^{*}$, we obtain

$$
\begin{aligned}
\Pi_{y_{0}}(f)(x) & =\sum_{k_{1} \in \mathcal{L}_{1}^{*}} \omega_{k_{1}}(x) \sum_{k_{2}:\left(k_{1}, k_{2}\right) \in \mathcal{L}^{*}} C\left(k_{1}, k_{2}\right) \int_{0}^{y_{0}} \omega_{k_{2}}(y) d y \\
& =\sum_{k_{1} \in \mathcal{L}_{1}^{*}} \omega_{k_{1}}(x) D\left(k_{1}\right),
\end{aligned}
$$

where $\mathcal{L}_{1}^{*}=\left\{k_{1}:\left(k_{1}, k_{2}\right) \in \mathcal{L}^{*}\right\}$ and $D\left(k_{1}\right)=\sum_{k_{2}:\left(k_{1}, k_{2}\right) \in \mathcal{L}^{*}} C\left(k_{1}, k_{2}\right) \int_{0}^{y_{0}} \omega_{k_{2}}(y) d y$. Note that the coefficients $D\left(k_{1}\right)$ depend on $y_{0}$.

The functions $\Pi_{y_{0}}(f)$ may be invariant under the action of some element $\left(v_{\alpha}, \alpha\right)$ of $\mathbf{E}(n) \cong \mathbf{R}^{n} \ltimes \mathbf{O}(n)$. For $f \in X_{\Gamma}$ this is equivalent to

$$
\sum_{k_{1} \in \mathcal{L}_{1}^{*}} \omega_{k_{1}}(x) D\left(k_{1}\right)=\sum_{k_{1} \in \mathcal{L}_{1}^{*}} \omega_{k_{1}}\left(\alpha^{-1} x\right) \omega_{k_{1}}\left(-\alpha^{-1} v_{\alpha}\right) D\left(k_{1}\right) .
$$

This equation imposes restrictions on the coefficients $D\left(k_{1}\right)$, see Lemma 7.1 below.

Summarising, the minimal requirements are that $X_{\Gamma}$ is a vector space of functions and that:

(1) $\Gamma$ is a $(n+1)$-dimensional crystallographic group with lattice $\mathcal{L}$, dual lattice $\mathcal{L}^{*}$ and point group $\mathbf{J}$,

(2) if $f \in X_{\Gamma}$ then:

(i) $f: \mathbf{R}^{n+1} \longrightarrow \mathbf{R}$ is $\Gamma$-invariant,

(ii) $f$ has a Fourier expansion in waves $\omega_{k}(x, y), k \in \mathcal{L}^{*}$,

(iii) the integral and the summation commute in the projection of $f$,

(3) $\operatorname{Re}\left(I_{k}\right), \operatorname{Im}\left(I_{k}\right) \in X_{\Gamma}$ for all $k \in \mathcal{L}^{*}$ with $I_{k}(x, y)=\sum_{\delta \in \mathbf{J}} \omega_{\delta k}(x, y) \omega_{\delta k}\left(-v_{\delta}\right)$.

As stated in the Introduction, these minimal requirements are satisfied, for instance, if $X_{\Gamma}$ is the space of all $\Gamma$-invariant functions of class $C^{1}$ : the Fourier expansion of a $C^{1}$ function $f$ converges absolutely to $f$ (see for instance Section 11 of Chapter 3 of Tolstov [19]), and therefore (2i)-(2iii) hold.

\section{Sufficiency OF CONDITIONS IN TheOREM 1.2}

Each one of the conditions (I), (II) and (III) of Theorem 1.2 is sufficient by basic properties of integrals:

Lemma 3.1. All functions in $\Pi_{y_{0}}\left(X_{\Gamma}\right)$ are invariant under the action of $\left(v_{\alpha}, \alpha\right) \in$ $\mathbf{R}^{n} \ltimes \mathbf{O}(n)$ if one of the following conditions holds:

(I) $\left(\left(v_{\alpha}, 0\right), \alpha_{+}\right) \in \Gamma$,

(II) $\left(\left(v_{\alpha}, y_{0}\right), \alpha_{-}\right) \in \Gamma$,

(III) $\left(0, y_{0}\right) \in \mathcal{L}$ and either $\left(\left(v_{\alpha}, y_{1}\right), \alpha_{+}\right) \in \Gamma$ or $\left(\left(v_{\alpha}, y_{1}\right), \alpha_{-}\right) \in \Gamma$, for some $y_{1} \in \mathbf{R}$.

Proof. Let $f$ be any function in $X_{\Gamma}$. If condition (I) holds then $f(x, y)=\left(\left(v_{\alpha}, 0\right), \alpha_{+}\right)$. $f(x, y)=f\left(\alpha^{-1} x-\alpha^{-1} v_{\alpha}, y\right)$ and so $\Pi_{y_{0}}(f)(x)=\int_{0}^{y_{0}} f(x, y) d y$ equals

$$
\int_{0}^{y_{0}} f\left(\alpha^{-1} x-\alpha^{-1} v_{\alpha}, y\right) d y=\Pi_{y_{0}}(f)\left(\alpha^{-1} x-\alpha^{-1} v_{\alpha}\right)=\left(v_{\alpha}, \alpha\right) \cdot \Pi_{y_{0}}(f)(x) .
$$

Similarly, if condition (II) holds then

$$
\Pi_{y_{0}}(f)(x)=\int_{0}^{y_{0}} f\left(\alpha^{-1} x-\alpha^{-1} v_{\alpha}, y_{0}-y\right) d y
$$


which, for $z=y_{0}-y$, equals

$$
\int_{0}^{y_{0}} f\left(\alpha^{-1} x-\alpha^{-1} v_{\alpha}, z\right) d z=\left(v_{\alpha}, \alpha\right) \cdot \Pi_{y_{0}}(f)(x) .
$$

If (III) holds then

$$
\Pi_{y_{0}}(f)(x)=\int_{0}^{y_{0}} f\left(\alpha^{-1} x-\alpha^{-1} v_{\alpha}, \pm\left(y-y_{1}\right)\right) d y
$$

which equals either $\int_{-y_{1}}^{y_{0}-y_{1}} f\left(\alpha^{-1} x-\alpha^{-1} v_{\alpha}, z\right) d z$ or $\int_{y_{1}-y_{0}}^{y_{1}} f\left(\alpha^{-1} x-\alpha^{-1} v_{\alpha}, z\right) d z$. Since $f$ has period $\left(0, y_{0}\right)$, both integrals are equal to

$$
\int_{0}^{y_{0}} f\left(\alpha^{-1} x-\alpha^{-1} v_{\alpha}, z\right) d z=\left(v_{\alpha}, \alpha\right) \cdot \Pi_{y_{0}}(f)(x) .
$$

\section{Symmetry of $\Pi_{y_{0}}\left(X_{\Gamma}\right)$ Related to $\Gamma$ and $\mathcal{L}^{*}$}

The proof that the conditions (I), (II) and (III) of Theorem 1.2 are necessary explores the restrictions on $\mathcal{L}^{*}$ imposed by the presence of $\left(v_{\alpha}, \alpha\right)$ in the group of symmetries of $\Pi_{y_{0}}\left(X_{\Gamma}\right)$. The restrictions will be characterised by subsets of $\mathcal{L}^{*}$.

Given $y_{0}>0 \in \mathbf{R}$ and $\alpha \in \mathbf{O}(n)$, if either $\left(v_{+}, \alpha_{+}\right)$or $\left(v_{-}, \alpha_{-}\right)$lies in $\Gamma$, let $\mathcal{M}^{*}$, $\mathcal{M}_{+}^{*}$ and $\mathcal{M}_{-}^{*}$ be the modules

$$
\mathcal{M}^{*}=\left\{k \in \mathcal{L}^{*}:<k, \sigma v_{+}-v_{+}>\in \mathbf{Z}\right\}
$$

$\mathcal{M}_{+}^{*}=\left\{k \in \mathcal{L}^{*}:<k, v_{+}-\left(v_{\alpha}, 0\right)>\in \mathbf{Z}\right\} \quad \mathcal{M}_{-}^{*}=\left\{k \in \mathcal{L}^{*}:<k, v_{-}-\left(v_{\alpha}, y_{0}\right)>\in \mathbf{Z}\right\}$.

If $\alpha_{+} \notin \mathbf{J}$ then $\mathcal{M}^{*}=\mathcal{M}_{+}^{*}=\{0\}$ and if $\alpha_{-} \notin \mathbf{J}$ then $\mathcal{M}_{-}^{*}=\{0\}$. Similarly, if any of $\left(v_{+}, \alpha_{+}\right),\left(v_{-}, \alpha_{-}\right)$or $\left(v_{\sigma}, \sigma\right)$ lies in $\Gamma$, let

$$
\begin{aligned}
& \mathcal{N}^{*}=\left\{k \in \mathcal{L}^{*}:<k, \sigma v_{+}-v_{+}>+\frac{1}{2} \in \mathbf{Z}\right\} \quad \mathcal{N}_{y_{0}}^{*}=\left\{k \in \mathcal{L}^{*}:<k,\left(0, y_{0}\right)>\in \mathbf{Z}-\{0\}\right\} \\
& \mathcal{N}_{\sigma}^{*}=\left\{k \in \mathcal{L}^{*}:<k, v_{\sigma}-\left(0, y_{0}\right)>+\frac{1}{2} \in \mathbf{Z}\right\} \quad \mathcal{N}_{\tilde{\sigma}}^{*}=\left\{k \in \mathcal{L}^{*}:<k, v_{\sigma}-\left(0, y_{0}\right)> \pm \frac{1}{4} \in \mathbf{Z}\right\} .
\end{aligned}
$$

If the defining element is not in $\Gamma$ we take the subset to be empty. The last four sets are not modules. The smallest modules containing them are

$$
\overline{\mathcal{N}^{*}}=\mathcal{N}^{*} \cup \mathcal{M}^{*} \quad \overline{\mathcal{N}_{y_{0}}^{*}}=\mathcal{N}_{y_{0}}^{*} \cup \mathcal{M}_{y_{0}}^{*} \quad \overline{\mathcal{N}_{\sigma}^{*}}=\mathcal{N}_{\sigma}^{*} \cup \mathcal{M}_{\sigma}^{*} \quad \overline{\mathcal{N}_{\tilde{\sigma}}^{*}}=\mathcal{N}_{\tilde{\sigma}}^{*} \cup \overline{\mathcal{N}_{\sigma}^{*}},
$$

where all the unions are disjoint, and $\mathcal{M}_{y_{0}}^{*}$ and $\mathcal{M}_{\sigma}^{*}$ are the modules

$\mathcal{M}_{y_{0}}^{*}=\left\{k \in \mathcal{L}^{*}:<k,\left(0, y_{0}\right)>=0\right\}$ and $\mathcal{M}_{\sigma}^{*}=\left\{k \in \mathcal{L}^{*}:<k, v_{\sigma}-\left(0, y_{0}\right)>\in \mathbf{Z}\right\}$.

These subsets do not depend on the choice of non-orthogonal components $v_{+}$, $v_{-}$and $v_{\sigma}$ : any element in the cosets $v_{+}+\mathcal{L}, v_{-}+\mathcal{L}$ and $v_{\sigma}+\mathcal{L}$ defines the same subsets.

Properties of $\mathcal{N}_{\sigma}^{*}$ and $\mathcal{N}_{\tilde{\sigma}}^{*}$. Let $m_{1}, m_{2} \in \mathbf{Z}$.

(1) If $g_{1}, g_{2} \in \mathcal{N}_{\sigma}^{*}$ then $m_{1} g_{1}+m_{2} g_{2} \in\left\{\begin{array}{ccc}\mathcal{M}_{\sigma}^{*} & \text { if } & m_{1}+m_{2} \text { even } \\ \mathcal{N}_{\sigma}^{*} & \text { if } & m_{1}+m_{2} \text { odd. }\end{array}\right.$

(2) If $g_{1}, g_{2} \in \mathcal{N}_{\tilde{\sigma}}^{*}$ then $m_{1} g_{1}+m_{2} g_{2} \in\left\{\begin{array}{llll}\overline{\mathcal{N}_{\sigma}^{*}} & \text { if } & m_{1}+m_{2} \text { even } \\ \mathcal{N}_{\tilde{\sigma}}^{*} & \text { if } & m_{1}+m_{2} \text { odd. }\end{array}\right.$

With this notation, the analogue of Theorem 1.2 for $\mathcal{L}^{*}$ takes the form:

Proposition 4.1. All functions in $\Pi_{y_{0}}\left(X_{\Gamma}\right)$ are invariant under the action of $\left(v_{\alpha}, \alpha\right) \in \mathbf{R}^{n} \ltimes \mathbf{O}(n)$ if and only if one of the following conditions holds:

(A) $\left(v_{+}, \alpha_{+}\right) \in \Gamma$ for some $v_{+} \in \mathbf{R}^{n+1}$ and $\mathcal{L}^{*}=\mathcal{N}_{y_{0}}^{*} \cup \mathcal{M}_{+}^{*}$,

(B) $\left(v_{-}, \alpha_{-}\right) \in \Gamma$ for some $v_{-} \in \mathbf{R}^{n+1}$ and $\mathcal{L}^{*}=\mathcal{N}_{y_{0}}^{*} \cup \mathcal{M}_{-}^{*}$, 
(C) both $\left(v_{\sigma}, \sigma\right)$ and $\left(v_{+}, \alpha_{+}\right) \in \Gamma$ for some $v_{\sigma}, v_{+} \in \mathbf{R}^{n+1}$ and, moreover, $\mathcal{M}^{*} \subset\left(\mathcal{N}_{y_{0}}^{*} \cup \mathcal{M}_{+}^{*} \cup \mathcal{N}_{\sigma}^{*}\right)$ and $\mathcal{N}^{*} \subset\left(\mathcal{N}_{y_{0}}^{*} \cup\left(\mathcal{M}_{-}^{*} \cap \mathcal{N}_{\tilde{\sigma}}^{*}\right)\right)$.

The proof that the conditions of Proposition 4.1 are necessary is postponed to Section 7. In Section 6 below we show that the conditions (A), (B) and (C) of Proposition 4.1 imply the cases (I), (II) and (III) of Theorem 1.2. Together with Lemma 3.1 this also establishes the sufficiency in the Proposition.

\section{Properties of $\Gamma, \mathcal{L}$ and $\mathcal{L}^{*}$}

In this section we establish some basic results that will be used in the sequel. The simultaneous presence of the reflection $\left(v_{\sigma}, \sigma\right)$ and of $\left(v_{+}, \alpha_{+}\right)$in a group $\Gamma$ imposes strong restrictions on $\mathcal{L}^{*}$. One of these restrictions is the subject of the next Lemma.

Lemma 5.1. If both $\left(v_{\sigma}, \sigma\right) \in \Gamma$ and $\left(v_{+}, \alpha_{+}\right) \in \Gamma$ then $2\left(\sigma v_{+}-v_{+}\right) \in \mathcal{L}$ and $\overline{\mathcal{N}^{*}}=\mathcal{L}^{*}$.

Proof. Since $\left(v_{\sigma}, \sigma\right) \cdot\left(v_{+}, \alpha_{+}\right)=\left(v_{\sigma}+\sigma v_{+}, \alpha_{-}\right)$and $\left(v_{+}, \alpha_{+}\right) \cdot\left(v_{\sigma}, \sigma\right)=\left(v_{+}+\right.$ $\left.\alpha_{+} v_{\sigma}, \alpha_{-}\right)$, then $v=v_{\sigma}+\sigma v_{+}-v_{+}-\alpha_{+} v_{\sigma} \in \mathcal{L}$. As $\sigma \mathcal{L}=\mathcal{L}$ then $v-\sigma v=2\left(\sigma v_{+}-\right.$ $\left.v_{+}\right)+\left(I d_{n+1}-\alpha_{+}-\sigma+\alpha_{-}\right) v_{\sigma}$ also belongs to $\mathcal{L}$. Using $-\alpha_{+}-\sigma+\alpha_{-}=-I d_{n+1}$ we get $v-\sigma v=2\left(\sigma v_{+}-v_{+}\right)$or, equivalently, $2<k, \sigma v_{+}-v_{+}>\in \mathbf{Z}$, for all $k \in \mathcal{L}^{*}$.

Lemma 5.2 (Properties of the bases for $\mathcal{L}$ and $\mathcal{L}^{*}$ and notation). Let $\left\{l_{1}, \ldots, l_{n+1}\right\}$ be a basis for $\mathcal{L}$ and $\left\{l_{1}^{*}, \ldots, l_{n+1}^{*}\right\}$ be its dual basis. The matrices $M$ with rows $l_{1}, \ldots, l_{n+1}$ and $M^{*}$ with rows $l_{1}^{*}, \ldots, l_{n+1}^{*}$, are related by $M^{*}=\left(M^{-1}\right)^{T}$, and satisfy:

(1) If $\left(v_{\delta}, \delta\right) \in \Gamma$ then, given the real numbers $r_{1}, \ldots, r_{n+1}$, there exists $l \in \mathcal{L}$ such that $v_{\delta}+l=\sum_{i=1}^{n+1} s_{i} l_{i}$ with $\left(s_{i}-r_{i}\right) \in[0,1[$ for all $i \in\{1, \ldots, n+1\}$.

(2) If $(0, a) \in \mathcal{L}$ for some $a \neq 0$ then we may choose the basis $\left\{l_{1}, \ldots, l_{n+1}\right\}$ for $\mathcal{L}$ such that

(i) $M=\left(\begin{array}{cc}A & B \\ 0 & b\end{array}\right)$ where $A$ is an $n \times n$ matrix with rows $a_{1}, \ldots, a_{n} \in \mathbf{R}^{n}$ and $B=\left(b_{1}, \ldots, b_{n}\right)^{T}$, with $b=\frac{a}{m}$ for some $m \in \mathbf{Z}$ and $b_{i} \in \mathbf{R}$.

(ii) $M^{*}=\left(\begin{array}{cc}A^{*} & 0 \\ -\frac{1}{b} B^{T} A^{*} & \frac{1}{b}\end{array}\right)$, where $A^{*}=\left(A^{-1}\right)^{T}$ has rows $a_{1}^{*}, \ldots, a_{n}^{*}$ with $<a_{i}^{*}, a_{j}>=\delta_{i j}$ for $i, j \in\{1, \ldots, n\}$.

(iii) The set $\left\{a_{1}, \ldots, a_{n}\right\}$ is a basis for a lattice in $\mathbf{R}^{n}$ and $\left\{a_{1}^{*}, \ldots, a_{n}^{*}\right\}$ is a basis for its dual.

(iv) $l_{i}^{*}=\left(a_{i}^{*}, 0\right)$ for $i \in\{1, \ldots, n\}$ and $\mathcal{M}_{y_{0}}^{*}=\left\{l_{1}^{*}, \ldots, l_{n}^{*}\right\}_{\mathbf{Z}}$.

(3) If $\sigma$ lies in the holohedry of $\mathcal{L}$ then $\mathcal{L}$ contains an element of the form $(0, a)$, $a \neq 0$. Moreover, each entry $b_{i}$ of $B$ may be taken to be either zero or $b / 2$.

Proof. (1) The set $\left\{l_{1}, \ldots, l_{n+1}\right\}$ is a basis for $\mathbf{R}^{n+1}$ and so $v_{\delta}=\sum_{i=1}^{n+1} \hat{s}_{i} l_{i}$ with $\hat{s}_{i} \in \mathbf{R}$ for all $i \in\{1, \ldots, n+1\}$. Denoting the integer part of $\hat{s}_{i}-r_{i}$ as $\left[\hat{s}_{i}-r_{i}\right]$, we may take $s_{i}=\hat{s}_{i}-\left[\hat{s}_{i}-r_{i}\right]$ and $l=\sum_{i=1}^{n+1}\left[\hat{s}_{i}-r_{i}\right] l_{i} \in \mathcal{L}$.

(2) Given $(0, a) \in \mathcal{L}, a \neq 0$, then $(0, b), b \neq 0$, the smallest element of $\mathcal{L}$ in the direction of $(0, a)$, is a generator, and $(0, a)=m(0, b)$ for some $m \in \mathbf{Z}$. Thus, there are elements $l_{1}, \ldots, l_{n}$ in $\mathcal{L}$ such that $\mathcal{L}=\left\{l_{1}, \ldots, l_{n},(0, b)\right\}_{\mathbf{z}}$. For $l_{i}=\left(a_{i}, b_{i}\right)$, with $i \in\{1, \ldots, n\}$, and $(0, b)=l_{n+1}$, we obtain the matrix $M$, and $M^{*}$ has the form given in (2ii). Property (2iv) follows from the definition of $\mathcal{M}_{y_{0}}^{*}$.

(3) For $(c, d)$ in $\mathcal{L}$ with $d \neq 0$, since $\sigma \mathcal{L}=\mathcal{L}$ then $(c, d)-\sigma(c, d)=(0,2 d) \in \mathcal{L}$ and property $(2)$ is valid. For $l_{i}=\left(a_{i}, b_{i}\right)$, with $i \in\{1, \ldots, n\}$, the elements $l_{i}-\sigma l_{i}=$ $\left(0,2 b_{i}\right)$ lie in $\mathcal{L}$ and so $\left(0,2 b_{i}\right)=m(0, b)$ for some $m \in \mathbf{Z}$. Therefore $l_{i}=\left(a_{i}, \frac{m b}{2}\right)$, and either $l_{i}=\left(a_{i}, 0\right)$ or $l_{i}=\left(a_{i}, \frac{b}{2}\right)$ up to multiples of $(0, b)=l_{n+1}$. 


\section{Proposition 4.1 implies Theorem 1.2}

Proposition 4.1 states that elements of $\Gamma$ ensuring symmetry after projection have orthogonal components $\alpha_{+}$or $\alpha_{-}$. Information on the non-orthogonal components $v_{+}, v_{-} \in \mathbf{R}^{n+1}$ appears as constraints on the structure of $\mathcal{L}^{*}$.

In this section we translate restrictions on $\Gamma$ and $\mathcal{L}^{*}$ into restrictions on $\Gamma$ and $\mathcal{L}$. The main tool will be to obtain information about a basis of $\mathcal{L}^{*}$ and use this to find a suitable basis for $\mathcal{L}$. Each condition of Proposition 4.1 is now treated in a separate lemma. We assume throughout that $\left(v_{\alpha}, \alpha\right) \in \Sigma_{y_{0}}$.

Lemma 6.1. If $\left(v_{+}, \alpha_{+}\right) \in \Gamma$ and $\mathcal{L}^{*}=\mathcal{N}_{y_{0}}^{*} \cup \mathcal{M}_{+}^{*}$ then one of the conditions holds: I. $\left(\left(v_{\alpha}, 0\right), \alpha_{+}\right) \in \Gamma$,

III. $\left(0, y_{0}\right) \in \mathcal{L}$ and $\left(\left(v_{\alpha}, y_{1}\right), \alpha_{+}\right) \in \Gamma$ for some $y_{1} \in \mathbf{R}$.

Proof. Since $\overline{\mathcal{N}_{y_{0}}^{*}} \subset \mathcal{N}_{y_{0}}^{*} \cup \mathcal{M}_{+}^{*}=\mathcal{L}^{*}$ and since both $\overline{\mathcal{N}_{y_{0}}^{*}}$ and $\mathcal{M}_{+}^{*}$ are modules, then either $\mathcal{L}^{*}=\overline{\mathcal{N}_{y_{0}}^{*}}$ or $\mathcal{L}^{*}=\mathcal{M}_{+}^{*}$. In the second case $\left\langle k, v_{+}-\left(v_{\alpha}, 0\right)>\in \mathbf{Z}\right.$ for all $k \in \mathcal{L}^{*}$, i.e. , $v_{+}-\left(v_{\alpha}, 0\right) \in \mathcal{L}$, and so (I) follows, because

$$
\left(-v_{+}+\left(v_{\alpha}, 0\right), I\right) \cdot\left(v_{+}, \alpha_{+}\right)=\left(\left(v_{\alpha}, 0\right), \alpha_{+}\right) \in \Gamma .
$$

If $\mathcal{L}^{*}=\overline{\mathcal{N}_{y_{0}}^{*}}$ then $\left(0, y_{0}\right) \in \mathcal{L}$. We may use the basis $\left\{l_{1}^{*}, \ldots, l_{n+1}^{*}\right\}$ for $\mathcal{L}^{*}$ with the properties (2) in Lemma 5.2. As $l_{n+1}^{*} \in \mathcal{N}_{y_{0}}^{*}$, it follows that $\frac{1}{b}=\frac{m}{y_{0}}, m \in \mathbf{Z}-\{0\}$. Also $l_{i}^{*} \in \mathcal{M}_{y_{0}}^{*} \subset \mathcal{M}_{+}^{*}$ for $i \in\{1, \ldots n\}$, hence $<l_{i}^{*}, v_{+}-\left(v_{\alpha}, 0\right)>\in \mathbf{Z}$. Any $k \in \mathcal{L}^{*}$ may be written as $k=k_{1}+m_{2} l_{n+1}^{*}$ with $k_{1} \in \mathcal{M}_{y_{0}}^{*}, m_{2} \in \mathbf{Z}$. For any $y_{1} \in \mathbf{R}$,

$$
\begin{aligned}
<k, v_{+}-\left(v_{\alpha}, y_{1}\right)> & =<k, v_{+}-\left(v_{\alpha}, 0\right)>-<k,\left(0, y_{1}\right)> \\
& =m_{1}+m_{2}<l_{n+1}^{*}, v_{+}-\left(v_{\alpha}, 0\right)>-m_{2} \frac{m}{y_{0}} y_{1},
\end{aligned}
$$

with $m_{1}, m_{2} \in \mathbf{Z}$. Taking $y_{1}=<l_{n+1}^{*}, v_{+}-\left(v_{\alpha}, 0\right)>\frac{y_{0}}{m}$ we get $<k, v_{+}-\left(v_{\alpha}, y_{1}\right)>\in$ Z. Thus, $v_{+}-\left(v_{\alpha}, y_{1}\right) \in \mathcal{L}$ for some $y_{1} \in \mathbf{R}$ and $\left(-v_{+}+\left(v_{\alpha}, y_{1}\right), I\right) \cdot\left(v_{+}, \alpha_{+}\right)=$ $\left(\left(v_{\alpha}, y_{1}\right), \alpha_{+}\right) \in \Gamma$, hence (III) holds.

Lemma 6.2. If $\left(v_{-}, \alpha_{-}\right) \in \Gamma$ and $\mathcal{L}^{*}=\mathcal{N}_{y_{0}}^{*} \cup \mathcal{M}_{-}^{*}$ then one of the conditions holds: II. $\left(\left(v_{\alpha}, y_{0}\right), \alpha_{-}\right) \in \Gamma$,

III. $\left(0, y_{0}\right) \in \mathcal{L}$ and $\left(\left(v_{\alpha}, y_{1}\right), \alpha_{-}\right) \in \Gamma$ for some $y_{1} \in \mathbf{R}$.

Proof. The proof is analogous to that of Lemma 6.1 with $v_{-}-\left(v_{\alpha}, y_{0}\right)$ instead of $v_{+}-\left(v_{\alpha}, 0\right)$ and $y_{1}=<l_{n+1}^{*}, v_{-}-\left(v_{\alpha}, y_{0}\right)>\frac{y_{0}}{m}+y_{0}$.

Lemma 6.3. If both $\left(v_{\sigma}, \sigma\right)$ and $\left(v_{+}, \alpha_{+}\right)$belong to $\Gamma$, and if both

$$
\mathcal{M}^{*} \subset\left(\mathcal{N}_{y_{0}}^{*} \cup \mathcal{M}_{+}^{*} \cup \mathcal{N}_{\sigma}^{*}\right) \text { and } \mathcal{N}^{*} \subset\left(\mathcal{N}_{y_{0}}^{*} \cup\left(\mathcal{M}_{-}^{*} \cap \mathcal{N}_{\tilde{\sigma}}^{*}\right)\right) \text {, }
$$

then one of the following conditions of Theorem 1.2 holds:

I. $\left(\left(v_{\alpha}, 0\right), \alpha_{+}\right) \in \Gamma$,

II. $\left(\left(v_{\alpha}, y_{0}\right), \alpha_{-}\right) \in \Gamma$,

III. $\left(0, y_{0}\right) \in \mathcal{L}$ and either $\left(\left(v_{\alpha}, y_{1}\right), \alpha_{+}\right) \in \Gamma$ or $\left(\left(v_{\alpha}, y_{1}\right), \alpha_{-}\right) \in \Gamma$, for some $y_{1} \in \mathbf{R}$.

The proof of Lemma 6.3 has three main steps. First, in Lemma 6.4 we obtain some properties of $\left(v_{\sigma}, \sigma\right)$ and $\left(v_{+}, \alpha_{+}\right)$and of the bases of $\mathcal{L}$ and $\mathcal{L}^{*}$. Writing $v_{+}=\left(v_{1}, v_{2}\right)$ with $v_{1} \in \mathbf{R}^{n}$ and $v_{2} \in \mathbf{R}$, we show that there are two possibilities for $v_{1}$, each one treated in a separate lemma.

Lemma 6.4. Under the conditions of Lemma 6.3, since $\sigma \in \mathbf{J}$, we may choose bases for $\mathcal{L}$ and $\mathcal{L}^{*}$ satisfying properties (1) to (3) in Lemma 5.2. In particular $(0, b) \in \mathcal{L}$ for some $b>0$. Moreover

(a) $v_{\sigma}+\sigma v_{\sigma} \in \mathcal{L}$.

(b) $\sigma v_{+}-v_{+}=-\left(0,2 v_{2}\right)$, hence $l_{i}^{*} \in \mathcal{M}^{*}$ for $i \in\{1, \ldots, n\}$. Therefore

(i) $\left(0,4 v_{2}\right) \in \mathcal{L}$ and

(ii) if $\left(0,2 v_{2}\right) \in \mathcal{L}$ then $\mathcal{N}^{*}=\emptyset$. 
(c) $l_{1}^{*}, \ldots, l_{n}^{*} \in \mathcal{M}_{y_{0}}^{*} \subset\left(\mathcal{M}_{+}^{*} \cup \mathcal{N}_{\sigma}^{*}\right)$.

(d) We may choose $v_{+}$so that either $v_{1}=v_{\alpha}$ or we may take $l_{1}=\left(a_{1}, b_{1}\right)$ with $a_{1}=2\left(v_{1}-v_{\alpha}\right)$.

(e) In both cases of property (d), $l_{i}^{*} \in \mathcal{M}_{+}^{*}$ for all $i \in\{2, \ldots, n\}$.

Proof. For (a), if $\left(v_{\sigma}, \sigma\right) \in \Gamma$ then $\left(v_{\sigma}, \sigma\right) \cdot\left(v_{\sigma}, \sigma\right)=\left(v_{\sigma}+\sigma v_{\sigma}, I\right) \in \Gamma$. For (b) we use Lemma 5.1. Then Lemma 5.2 implies that for all $i \in\{1, \ldots, n\}$ we have $l_{i}^{*}=\left(a_{i}^{*}, 0\right) \in \mathcal{M}_{y_{0}}^{*} \subset \mathcal{M}^{*}$ and (bi) and (bii) follow. Since $\mathcal{M}_{y_{0}}^{*} \subset \mathcal{M}^{*}$ and $\mathcal{M}_{y_{0}}^{*} \cap \mathcal{N}_{y_{0}}^{*}=\emptyset$, the hypothesis of Lemma 6.3 implies (c).

It follows that for all $i \in\{1, \ldots, n\}$, either $<\left(a_{i}^{*}, 0\right), v_{+}-\left(v_{\alpha}, 0\right)>\in \mathbf{Z}$ or $<\left(a_{i}^{*}, 0\right), v_{\sigma}-\left(0, y_{0}\right)>+\frac{1}{2} \in \mathbf{Z}$. If $l_{i}^{*} \in \mathcal{N}_{\sigma}^{*}$ then $2 l_{i}^{*} \notin \mathcal{N}_{\sigma}^{*}$ and so,

$$
2<\left(a_{i}^{*}, 0\right), v_{+}-\left(v_{\alpha}, 0\right)>=<a_{i}^{*}, 2\left(v_{1}-v_{\alpha}\right)>\in \mathbf{Z}, \quad i \in\{1, \ldots, n\},
$$

therefore, $2\left(v_{1}-v_{\alpha}\right)=\sum_{i=1}^{n} m_{i} a_{i}$ with $m_{i} \in \mathbf{Z}$ for $i \in\{1, \ldots, n\}$. If $v_{1} \neq v_{\alpha}$, we may choose $a_{1}=\frac{2\left(v_{1}-v_{\alpha}\right)}{m}$ for some $m \in \mathbf{Z}$, by the property (2iii) in Lemma 5.2. If $v_{\alpha}=\sum_{i=1}^{n} r_{i} a_{i}$, with $r_{i} \in \mathbf{R}$, by property (1) in Lemma 5.2 , there exists $(c, d) \in \mathcal{L}$ such that $\left(v_{1}+c, v_{2}+d\right)=\sum_{i=1}^{n+1} s_{i} l_{i}$ with $2\left(r_{i}-s_{i}\right) \in[0,2[$ for $i \in\{1, \ldots, n\}$. Note that $\mathcal{N}_{\sigma}^{*}$ does not depend on $v_{+}$and that $\left(v_{1}, v_{2}\right)$ and $\left(v_{1}+c, v_{2}+d\right)$ define the same set $\mathcal{M}_{+}^{*}$, thus $2\left(v_{1}+c-v_{\alpha}\right)=\sum_{i=1}^{n} \hat{m}_{i} a_{i}$ with $\hat{m}_{i} \in \mathbf{Z}$. Since $2\left(s_{1}-r_{1}\right)=\hat{m}_{1} \in \mathbf{Z}$ then $\hat{m}_{1}$ is either 0 or 1 and $(\mathrm{d})$ follows, replacing $v_{+}$by $v_{+}+(c, d)$. From now on we take this choice of $v_{+}$.

For (e) notice that $v_{1}-v_{\alpha}$ is either zero or $a_{1} / 2$. Therefore, for $i \in\{2, \ldots, n\}$ we have $<l_{i}^{*}, v_{+}-\left(v_{\alpha}, 0\right)>=<a_{i}^{*}, v_{1}-v_{\alpha}>=0$.

The two cases of Property (d) above are now treated in separate Lemmas.

Lemma 6.5. Under the conditions of Lemma 6.3 if $v_{1}=v_{\alpha}$ then one of the conditions of Theorem 1.2 holds.

Proof. If $v_{1}=v_{\alpha}$, then $\left(v_{\sigma}, \sigma\right) \cdot\left(v_{+}, \alpha_{+}\right)=\left(v_{-}, \alpha_{-}\right) \in \Gamma$ for $v_{-}=v_{\sigma}+\sigma v_{+}$, hence

$$
v_{-}-\left(v_{\alpha}, y_{0}\right)=v_{\sigma}-\left(0, v_{2}+y_{0}\right)
$$

Also $v_{+}-\left(v_{\alpha}, 0\right)=\left(0, v_{2}\right)$ and $l_{1}^{*}, \ldots, l_{n}^{*}$ lie in $\mathcal{M}^{*}$.

From Lemma $5.2(2 \mathrm{iv})$ and Lemma 6.4 it follows that for $i \in\{1, \ldots, n\}$ we have $<l_{i}^{*}+l_{n+1}^{*}, v>=<l_{n+1}^{*}, v>$ for $v \in\left\{v_{+}-\left(v_{\alpha}, 0\right),\left(0, y_{0}\right), \sigma v_{+}-v_{+}\right\}$, hence

$$
l_{i}^{*}+l_{n+1}^{*} \in \mathcal{Q}^{*} \quad \Leftrightarrow \quad l_{n+1}^{*} \in \mathcal{Q}^{*} \quad i \in\{1, \ldots, n\}
$$

where $\mathcal{Q}^{*}$ stands for any one of $\mathcal{M}_{+}^{*}, \mathcal{N}_{y_{0}}^{*}, \mathcal{N}^{*}$ or $\mathcal{M}^{*}$.

From property (b) in Lemma $6.4, l_{n+1}=(0, b)$ with $b=\frac{4 v_{2}}{m}$ for some $m \in \mathbf{Z}$. Therefore, $l_{n+1}^{*} \in \mathcal{M}_{+}^{*}$ if and only if $m=0(\bmod 4)$. In this case $\mathcal{L}^{*}=\mathcal{M}_{+}^{*}$ and hence $\left(0, v_{2}\right) \in \mathcal{L}$ and $\left(\left(v_{\alpha}, 0\right), \alpha_{+}\right) \in \Gamma$ as in Lemma 6.1 , i.e., condition (I) holds.

If $m=2(\bmod 4)$ then $l_{n+1}^{*} \in \mathcal{M}^{*}$. Since $l_{n+1}^{*} \notin \mathcal{M}_{+}^{*}$, the hypothesis of Lemma 6.3 implies that $l_{n+1}^{*}$ lies either in $\mathcal{N}_{y_{0}}^{*}$ or in $\mathcal{N}_{\sigma}^{*}$.

If $l_{n+1}^{*} \in \mathcal{N}_{y_{0}}^{*}$ then, by $(2) \mathcal{L}^{*}=\overline{\mathcal{N}_{y_{0}}^{*}}$ and this implies $\left(0, y_{0}\right) \in \mathcal{L}$. Condition (III) follows since $\left(\left(v_{\alpha}, v_{2}\right), \alpha_{+}\right) \in \Gamma$.

Now suppose that $l_{n+1}^{*} \notin\left(\mathcal{M}_{+}^{*} \cup \mathcal{N}_{y_{0}}^{*}\right)$, then by $(2) l_{i}^{*}+l_{n+1}^{*} \notin\left(\mathcal{M}_{+}^{*} \cup \mathcal{N}_{y_{0}}^{*}\right)$ for $i \in\{1, \ldots, n\}$ and thus $l_{i}^{*}+l_{n+1}^{*} \in \mathcal{N}_{\sigma}^{*}$. Let $k$ be either $k=l_{n+1}^{*}$ or $k=l_{i}^{*}+l_{n+1}^{*}$, then since $k \in \mathcal{N}_{\sigma}^{*}$, using (1) it follows that $k \in \mathcal{M}_{-}^{*}$ because

$$
<k, v_{-}-\left(v_{\alpha}, y_{0}\right)>=<k, v_{\sigma}-\left(0, y_{0}\right)>-<l_{n+1}^{*},\left(0, v_{2}\right)>\in \mathbf{Z} .
$$

Thus $\mathcal{L}^{*}=\mathcal{M}_{-}^{*}$ implying $v_{-}-\left(v_{\alpha}, y_{0}\right) \in \mathcal{L}$ and $\left(v_{-}-\left(v_{-}-\left(v_{\alpha}, y_{0}\right)\right), \alpha_{-}\right)=$ $\left(\left(v_{\alpha}, y_{0}\right), \alpha_{-}\right) \in \Gamma$, i.e. condition (II). 
If $m= \pm 1(\bmod 4)$ then $l_{n+1}^{*} \in \mathcal{N}^{*} \subset \mathcal{N}_{y_{0}}^{*} \cup\left(\mathcal{M}_{-}^{*} \cap \mathcal{N}_{\sigma}^{*}\right)$ and, using (2), $l_{i}^{*}+$ $l_{n+1}^{*} \in \mathcal{N}^{*}$ for $i \in\{1, \ldots, n\}$. If $l_{n+1}^{*} \in \mathcal{N}_{y_{0}}^{*}$ then condition (III) follows, as above.

Suppose $l_{n+1}^{*} \notin \mathcal{N}_{y_{0}}^{*}$ and hence, by $(2), l_{i}^{*}+l_{n+1}^{*} \notin \mathcal{N}_{y_{0}}^{*}$ for $i \in\{1, \ldots, n\}$. Thus $l_{i}^{*}+l_{n+1}^{*}$ and $l_{n+1}^{*}$ lie in $\mathcal{M}_{-}^{*}$ and hence $\mathcal{M}_{-}^{*}=\mathcal{L}^{*}$ implying condition (II).

Lemma 6.6. Under the conditions of Lemma 6.3 if $l_{1}=\left(2\left(v_{1}-v_{\alpha}\right), b_{1}\right)$ then one of the conditions of Theorem 1.2 holds.

Proof. When $v_{1} \neq v_{\alpha}$, assertion (2) in the proof of Lemma 6.5 holds when $\mathcal{Q}^{*}$ is any one of $\mathcal{N}_{y_{0}}^{*}, \mathcal{N}^{*}$ or $\mathcal{M}^{*}$. Since $l_{1}^{*} \notin \mathcal{M}_{+}^{*}$, from (c) in Lemma 6.4 we get $l_{1}^{*} \in \mathcal{N}_{\sigma}^{*}$.

Without loss of generality we may assume that $l_{i}^{*} \in \mathcal{M}_{\sigma}^{*}$ for $i \in\{2, \ldots, n\}$. To see this, note that $v_{\sigma}+\sigma v_{\sigma} \in \mathcal{L}$, hence $<l_{i}^{*}, v_{\sigma}+\sigma v_{\sigma}>\in \mathbf{Z}$. Using (2ii) in Lemma 5.2 it follows that $2<l_{i}^{*}, v_{\sigma}>\in \mathbf{Z}$ for $i \in\{2, \ldots, n\}$. If $l_{i}^{*} \in \mathcal{N}_{\sigma}^{*}$ for $i \in\left\{2, \ldots, n_{1}\right\}$, then $\tilde{l}_{i}^{*}=l_{i}^{*}-l_{1}^{*} \in \mathcal{M}_{\sigma}^{*}$. Let $\tilde{l}_{i}^{*}=l_{i}^{*}$ for $i=1$ and $i>n_{1}$, and $\tilde{l}_{1}=l_{1}-\sum_{i=2}^{n_{1}} l_{i}$, then $\left\{\tilde{l}_{i}^{*}\right\}$ is a basis for $\mathcal{L}^{*}$, the dual of the basis $\left\{\tilde{l}_{1}, l_{2}, \ldots, l_{n+1}\right\}$. The construction of $v_{+}$in the proof of (d) in Lemma 6.4 may be repeated for this basis, to get $\tilde{l}_{1}=\left(\tilde{a}_{1}, \tilde{b}_{1}\right)$ with $\tilde{a}_{1}=2\left(v_{1}-v_{\alpha}\right)$, establishing the claim.

Therefore $v_{\sigma}+l=\sum_{i=1}^{n+1} s_{i} l_{i}$ for some $l \in \mathcal{L}$, with $s_{1}=1 / 2$ and $s_{i}=0$ for $i \in\{2, \ldots, n\}$, so we may replace $v_{\sigma}$ by $v_{\sigma}+l$. Then $v_{\sigma}=\left(a_{1} / 2, b_{1} / 2\right)+s_{n+1}(0, b)$ and hence $v_{\sigma}+\sigma v_{\sigma}=\left(a_{1}, 0\right) \in \mathcal{L}$ implies $\left(0, b_{1}\right) \in \mathcal{L}$. Using the construction of Lemma $5.2(3)$ we get $b_{1}=0$ and

$$
v_{\sigma}=\frac{1}{2}\left(a_{1}, 0\right)+s_{n+1}(0, b)=v_{+}-\left(v_{\alpha}, 0\right)+\left(0, s_{n+1} b-v_{2}\right) .
$$

If $l_{n+1}^{*} \in \mathcal{N}_{y_{0}}^{*}$ then $\left(0, y_{0}\right) \in \mathcal{L}$. Then (3) implies that $v_{\sigma}-\left(a_{1}, 0\right)=-\sigma v_{+}+$ $\left(v_{\alpha}, s_{n+1} b-v_{2}\right)$. Condition (III) follows from

$$
\left(-\sigma v_{+}+\left(v_{\alpha}, s_{n+1} b-v_{2}\right), \sigma\right) \cdot\left(v_{+}, \alpha_{+}\right)=\left(\left(v_{\alpha}, s_{n+1} b-v_{2}\right), \alpha_{-}\right) \in \Gamma .
$$

Now suppose that $l_{n+1}^{*} \notin \mathcal{N}_{y_{0}}^{*}$. For $b=\frac{4 v_{2}}{m}$ we get $\left\langle l_{n+1}^{*}, \sigma v_{+}-v_{+}>=-\frac{2 v_{2}}{b}=\right.$ $-\frac{m}{2}$. If $m=2(\bmod 4)$ then $l_{n+1}^{*} \in \mathcal{M}^{*}$ and $l_{n+1}^{*} \notin \mathcal{M}_{+}^{*}$. Since we are assuming $l_{n+1}^{*} \notin \mathcal{N}_{y_{0}}^{*}$, then $l_{n+1}^{*} \in \mathcal{N}_{\sigma}^{*}$, where $s_{n+1}+y_{0} / b+1 / 2 \in \mathbf{Z}$ and, up to multiples of $(0, b), s_{n+1} b-v_{2}=y_{0}$. Condition (II) follows from (4).

For $m \neq 2(\bmod 4)$ with $l_{n+1}^{*} \notin \mathcal{N}_{y_{0}}^{*}$ the proof of the corresponding cases in Lemma 6.5 applies without any changes.

\section{Proof of necessity in Proposition 4.1}

We will show that if the hypothesis of Proposition 4.1 holds for the projection of the real and imaginary parts of $I_{k}(x, y)$, then one of the three conditions $(\mathrm{A}),(\mathrm{B})$ or $(\mathrm{C})$ must hold. In this section we identify all elements of the form $(v+l, \delta) \in \Gamma$, $l \in \mathcal{L}$, and denote them all by $\left(v_{\delta}, \delta\right)$, since $v_{\delta}$ and $v_{\delta}+l$ give rise to the same subsets of $\mathcal{L}^{*}$ defined in Section 4 and $w_{k}(v)=w_{k}(v+l)$ for $k \in \mathcal{L}^{*}$ and $l \in \mathcal{L}$.

For $\delta \in \mathbf{O}(n+1)$ and $k \in \mathcal{L}^{*}$, let $\delta k=\left(\tilde{k}_{1}, \tilde{k}_{2}\right)$, where $\tilde{k}_{1} \in \mathbf{R}^{n}$ and $\tilde{k}_{2} \in \mathbf{R}$. With the notation $\left.\delta k\right|_{1}=\tilde{k}_{1}$ and $\left.\delta k\right|_{2}=\tilde{k}_{2}$, let $\mathrm{J}^{I d}(k)$ be the subset of $\mathbf{J}$ that preserves $k_{1}$ given by

$$
\mathrm{J}^{I d}(k)=\left\{\delta \in \mathbf{J}:\left.\delta k\right|_{1}=k_{1}\right\}=\{\delta \in \mathbf{J}: \delta k=k \vee \delta k=\sigma k\}
$$

where the second equality follows by orthogonality of $\mathbf{J}$, since any element of the orbit $\mathbf{J}\left(k_{1}, k_{2}\right)$ whose $n$ first components equal $k_{1}$ is of the form $\left(k_{1}, \pm k_{2}\right)$. Let

$$
\mathbf{J}^{\alpha}(k)=\left\{\delta \in \mathbf{J}:\left.\delta k\right|_{1}=\alpha^{-1} k_{1}\right\}=\left\{\delta \in \mathbf{J}: \delta k=\alpha_{+}^{-1} k \vee \delta k=\alpha_{-}^{-1} k\right\}
$$

where the two expressions for $\mathbf{J}^{\alpha}(k)$ are equivalent because the elements on $\mathbf{J}\left(k_{1}, k_{2}\right)$ with $n$ first components $\alpha^{-1} k_{1}$ are of the form $\left(\alpha^{-1} k_{1}, \pm k_{2}\right)$, by orthogonality of $\mathbf{J}$ and of $\alpha$. Let $\alpha \mathcal{L}_{1}^{*}=\left\{\alpha k_{1}: k_{1} \in \mathcal{L}_{1}^{*}\right\}$. 
Lemma 7.1. If $\left(v_{\alpha}, \alpha\right) \in \Sigma_{y_{0}}$ then for all $k=\left(k_{1}, k_{2}\right) \in \mathcal{L}^{*}$, the following invariance conditions hold:

(a) if $k_{1} \in \mathcal{L}_{1}^{*} \cap \alpha \mathcal{L}_{1}^{*}$ then $\sum_{\delta \in \mathrm{J}^{I d}(k)} D^{\prime}(\delta, k)-\omega_{k_{1}}\left(-v_{\alpha}\right) \sum_{\delta \in \mathrm{J}^{\alpha}(k)} D^{\prime}(\delta, k)=0$

(b) if $k_{1} \notin \mathcal{L}_{1}^{*} \cap \alpha \mathcal{L}_{1}^{*}$ then $\sum_{\delta \in \mathrm{J}^{I d}(k)} D^{\prime}(\delta, k)=0$

where $D^{\prime}(\delta, k)=\omega_{\delta k}\left(-v_{\delta}\right) \int_{0}^{y_{0}} \omega_{\left.\delta k\right|_{2}}(y) d y$.

Proof. Notice first that for any $f \in X_{\Gamma}$ the equality

$$
\Pi_{y_{0}}(f)(x)=\left(v_{\alpha}, \alpha\right) \cdot \Pi_{y_{0}}(f)(x)=\Pi_{y_{0}}(f)\left(\alpha^{-1} x-\alpha^{-1} v_{\alpha}\right)
$$

is equivalent to

$$
\sum_{k_{1} \in \mathcal{L}_{1}^{*}} \omega_{k_{1}}(x) D\left(k_{1}\right)=\sum_{k_{1} \in \mathcal{L}_{1}^{*}} \omega_{k_{1}}\left(\alpha^{-1} x\right) \omega_{k_{1}}\left(-\alpha^{-1} v_{\alpha}\right) D\left(k_{1}\right),
$$

where, by orthogonality, the right hand side equals $\sum_{k_{1} \in \mathcal{L}_{1}^{*}} \omega_{\alpha k_{1}}(x) \omega_{\alpha k_{1}}\left(-v_{\alpha}\right) D\left(k_{1}\right)$ and, for $\tilde{k}_{1}=\alpha k_{1}$, is given by $\sum_{\tilde{k}_{1} \in \alpha \mathcal{L}_{1}^{*}} \omega_{\tilde{k}_{1}}(x) \omega_{\tilde{k}_{1}}\left(-v_{\alpha}\right) D\left(\alpha^{-1} \tilde{k}_{1}\right)$. Thus, from $\left(v_{\alpha}, \alpha\right) \in \Sigma_{y_{0}}$ it follows that if $k_{1} \notin \mathcal{L}_{1}^{*} \cap \alpha \mathcal{L}_{1}^{*}$ then $D\left(k_{1}\right)=0$ and if $k_{1} \in \mathcal{L}_{1}^{*} \cap \alpha \mathcal{L}_{1}^{*}$ then $D\left(k_{1}\right)=\omega_{k_{1}}\left(-v_{\alpha}\right) D\left(\alpha^{-1} k_{1}\right)$.

Each one of the functions $I_{k}$ is given by a summation over a $\mathbf{J}$-orbit on $\mathcal{L}^{*}$. Writing its projection in the form $\Pi_{y_{0}}\left(I_{k}\right)(x)=\sum_{\tilde{k}_{1} \in \mathcal{L}_{1}^{*}} \omega_{\tilde{k}_{1}}(x) D\left(\tilde{k}_{1}\right)$ we have $D\left(\tilde{k}_{1}\right)=0$ except for $\tilde{k}_{1}=\left.\delta k\right|_{1}$ when it is

$$
D\left(\tilde{k}_{1}\right)=\sum_{\delta \in \mathbf{J}:\left.\delta k\right|_{1}=\tilde{k}_{1}} \omega_{\delta k}\left(v_{\delta}\right) \int_{0}^{y_{0}} \omega_{\left.\delta k\right|_{2}}(y) d y .
$$

In particular, for $\tilde{k}_{1}=k_{1}$ the coefficient is $D\left(k_{1}\right)=\sum_{\delta \in J^{I d}(k)} D^{\prime}(\delta, k)$. If $k_{1} \notin$ $\mathcal{L}_{1}^{*} \cap \alpha \mathcal{L}_{1}^{*}$ this establishes condition (b).

If $k_{1} \in \mathcal{L}_{1}^{*} \cap \alpha \mathcal{L}_{1}^{*}$ we use $D\left(\alpha^{-1} k_{1}\right)=\sum_{\delta \in J^{\alpha}(k)} \omega_{\delta k}\left(v_{\delta}\right) \int_{0}^{y_{0}} \omega_{\left.\delta k\right|_{2}}(y) d y$, and condition (a) follows.

Although the invariance conditions in Lemma 7.1 involve the sets $\mathrm{J}^{I d}(k)$ and $\mathrm{J}^{\alpha}(k)$ for all $k \in \mathcal{L}^{*}$, we will show that for this proof we will only need to consider the sets:

$$
\mathbf{J}^{I d}=\left\{I d_{n+1}, \sigma\right\} \cap \mathbf{J} \text { and } \mathbf{J}^{\alpha}=\left\{\alpha_{+}^{-1}, \alpha_{-}^{-1}\right\} \cap \mathbf{J} .
$$

The rest of the proof is divided in three Lemmas. In Lemma 7.2 we describe all the possibilities for $\mathbf{J}^{I d}$ and $\mathbf{J}^{\alpha}$ and obtain in each case some consequences for $\mathcal{L}^{*}$ in terms of the subsets defined before the statement of Proposition 4.1. In Lemma 7.3 we study the set of all $k \in \mathcal{L}^{*}$ such that either $\mathrm{J}^{I d}(k) \neq \mathbf{J}^{I d}$ or $\mathrm{J}^{\alpha}(k) \neq \mathbf{J}^{\alpha}$. Finally, conditions (A), (B) and (C) are obtained in Lemma 7.4.

The next lemma describes, under the hypothesis of Proposition 4.1, the set

$$
\mathcal{O}^{*}=\left\{k \in \mathcal{L}^{*}: \mathbf{J}^{I d}(k)=\mathbf{J}^{I d} \wedge \mathbf{J}^{\alpha}(k)=\mathbf{J}^{\alpha}\right\}
$$

according to each of the cases for $\mathbf{J}^{I d}$ and $\mathbf{J}^{\alpha}$. This allows us to restate the invariance conditions (a) and (b) in simpler form in terms of subsets of $\mathcal{L}^{*}$.

Lemma 7.2. Suppose that the invariance conditions (a) and (b) hold for all $k=$ $\left(k_{1}, k_{2}\right) \in \mathcal{L}^{*}$. Then we have one of the following cases:

(1) $\mathbf{J}^{I d}=\left\{I d_{n+1}\right\}, \mathbf{J}^{\alpha}=\emptyset$ and $\mathcal{O}^{*} \subset \mathcal{N}_{y_{0}}^{*}$,

(2) $\mathbf{J}^{I d}=\left\{I d_{n+1}, \sigma\right\}, \mathbf{J}^{\alpha}=\emptyset$ and $\mathcal{O}^{*} \subset\left(\mathcal{N}_{y_{0}}^{*} \cup \mathcal{N}_{\sigma}^{*}\right)$,

(3) $\mathbf{J}^{I d}=\left\{I d_{n+1}\right\}, \mathbf{J}^{\alpha}=\left\{\alpha_{+}^{-1}\right\}$ and $\mathcal{O}^{*} \subset\left(\mathcal{N}_{y_{0}}^{*} \cup \mathcal{M}_{+}^{*}\right)$,

(4) $\mathbf{J}^{I d}=\left\{I d_{n+1}\right\}, \mathbf{J}^{\alpha}=\left\{\alpha_{-}^{-1}\right\}$ and $\mathcal{O}^{*} \subset\left(\mathcal{N}_{y_{0}}^{*} \cup \mathcal{M}_{-}^{*}\right)$,

(5) $\mathbf{J}^{I d}=\left\{I d_{n+1}, \sigma\right\}, \mathbf{J}^{\alpha}=\left\{\alpha_{+}^{-1}, \alpha_{-}^{-1}\right\}$,

$\left(\mathcal{O}^{*} \cap \mathcal{M}^{*}\right) \subset\left(\mathcal{N}_{y_{0}}^{*} \cup \mathcal{M}_{+}^{*} \cup \mathcal{N}_{\sigma}^{*}\right)$ and $\left(\mathcal{O}^{*} \cap \mathcal{N}^{*}\right) \subset\left(\mathcal{N}_{y_{0}}^{*} \cup\left(\mathcal{M}_{-}^{*} \cap \mathcal{N}_{\widetilde{\sigma}}^{*}\right)\right)$. 
Proof. Cases (1-5) enumerate all the possibilities for $\mathbf{J}^{I d}$ and $\mathbf{J}^{\alpha}$. This happens because $\mathbf{J}^{I d}$ is a group; if $\alpha_{+}^{-1}, \alpha_{-}^{-1} \in \mathbf{J}$ then $\alpha_{+} \alpha_{-}^{-1}=\sigma \in \mathbf{J}$ and if $\sigma \in \mathbf{J}$ then either $\mathbf{J}^{\alpha}=\emptyset$ or $\mathbf{J}^{\alpha}$ has two elements.

If $\left(v_{\sigma}, \sigma\right) \in \Gamma$ then $v_{\sigma}+\sigma v_{\sigma} \in \mathcal{L}$ as in Lemma 6.4 (a) and therefore we have

$$
\omega_{k}\left(-\sigma v_{\sigma}\right)=\omega_{k}\left(v_{\sigma}\right) \quad \text { if } k \in \mathcal{L}^{*} \text { and }\left(v_{\sigma}, \sigma\right) \in \Gamma \text {. }
$$

If $\mathbf{J}^{\alpha}=\emptyset$ then, for all $k=\left(k_{1}, k_{2}\right) \in \mathcal{O}^{*}$, the conditions in the hypothesis of the lemma become $\sum_{\delta \in \mathbf{J}^{I d}} D^{\prime}(\delta, k)=0$. In case (1) this means that $\int_{0}^{y_{0}} \omega_{k_{2}}(y) d y=0$ and hence $k \in \mathcal{N}_{y_{0}}^{*}$. In case (2) we have $\int_{0}^{y_{0}} \omega_{k_{2}}(y) d y+\omega_{\sigma k}\left(-v_{\sigma}\right) \int_{0}^{y_{0}} \omega_{-k_{2}}(y) d y=0$. Using

$$
\int_{0}^{y_{0}} \omega_{-k_{2}}(y) d y=\omega_{k_{2}}\left(-y_{0}\right) \int_{0}^{y_{0}} \omega_{k_{2}}(y) d y
$$

this is equivalent to $\left(1+\omega_{k}\left(v_{\sigma}-\left(0, y_{0}\right)\right)\right) \int_{0}^{y_{0}} \omega_{k_{2}}(y) d y=0$, by property (8) and the result follows because $1+\omega_{k}\left(v_{\sigma}-\left(0, y_{0}\right)\right)=0$ implies $k \in \mathcal{N}_{\sigma}^{*}$.

In the remaining cases either $\left(v_{+}, \alpha_{+}\right)$or $\left(v_{-}, \alpha_{-}\right)$belongs to $\Gamma$. Thus, $\alpha \mathcal{L}_{1}^{*}=\mathcal{L}_{1}^{*}$ and the first condition in the hypothesis of the lemma must be verified for all $k_{1} \in \mathcal{L}_{1}^{*}$. For $k \in \mathcal{O}^{*}$ this condition becomes

$$
\sum_{\delta \in \mathbf{J}^{I d}} D^{\prime}(\delta, k)-\omega_{k_{1}}\left(-v_{\alpha}\right) \sum_{\delta \in \mathbf{J}^{\alpha}} D^{\prime}(\delta, k)=0 .
$$

The proof for these cases consists of using properties (8) and (9) to rewrite condition (10) in the form $G\left(k_{1}, k_{2}\right) \int_{0}^{y_{0}} \omega_{k_{2}}(y) d y=0$ for $k \in \mathcal{O}^{*}$. This implies that either $k \in \mathcal{N}_{y_{0}}^{*}$ as in case $(1)$, or $G\left(k_{1}, k_{2}\right)=0$.

In case (3), this condition is $G\left(k_{1}, k_{2}\right)=\left(1-\omega_{k_{1}}\left(-v_{\alpha}\right) \omega_{k}\left(v_{+}\right)\right)=0$ implying $k \in$ $\mathcal{M}_{+}^{*}$. For case (4) it takes the form $G\left(k_{1}, k_{2}\right)=\left(1-\omega_{k_{1}}\left(-v_{\alpha}\right) \omega_{k}\left(v_{-}\right) \omega_{k_{2}}\left(-y_{0}\right)\right)=0$, which implies $k \in \mathcal{M}_{-}^{*}$. In case (5) we get

$$
2 G\left(k_{1}, k_{2}\right)=1+\omega_{k}\left(v_{\sigma}\right) \omega_{k_{2}}\left(-y_{0}\right)-\omega_{k_{1}}\left(-v_{\alpha}\right)\left(\omega_{k}\left(v_{+}\right)+\omega_{k}\left(v_{-}\right) \omega_{k_{2}}\left(-y_{0}\right)\right)=0 .
$$

Using Lemma 5.1 we get $\mathcal{O}^{*} \subset\left(\mathcal{M}^{*} \cup \mathcal{N}^{*}\right)$ and

$$
\omega_{k}\left(v_{-}\right)=\omega_{k}\left(v_{\sigma}\right) \omega_{k}\left(\sigma v_{+}\right) .
$$

If $k=\left(k_{1}, k_{2}\right) \in \mathcal{M}^{*}$ then $G\left(k_{1}, k_{2}\right)=0$ is equivalent to

$$
\begin{gathered}
1+\omega_{k}\left(v_{\sigma}\right) \omega_{k_{2}}\left(-y_{0}\right)-\omega_{k_{1}}\left(-v_{\alpha}\right) \omega_{k}\left(v_{+}\right)\left(1+\omega_{k}\left(\sigma v_{+}-v_{+}\right) \omega_{k}\left(v_{\sigma}\right) \omega_{k_{2}}\left(-y_{0}\right)\right) \\
=\left(1-\omega_{k}\left(v_{+}-\left(v_{\alpha}, 0\right)\right)\right)\left(1+\omega_{k}\left(v_{\sigma}-\left(0, y_{0}\right)\right)\right)=0
\end{gathered}
$$

and the result follows. For $k=\left(k_{1}, k_{2}\right) \in \mathcal{N}^{*}$, then $\omega_{k}\left(\sigma v_{+}\right) \omega_{k}\left(-v_{+}\right)=-1$ and

$$
\begin{aligned}
\omega_{k_{1}}\left(-v_{\alpha}\right) \omega_{k}\left(v_{+}\right) & =-\omega_{k_{1}}\left(-v_{\alpha}\right) \omega_{k}\left(\sigma v_{+}\right) \\
& =-\omega_{k_{1}}\left(-v_{\alpha}\right) \omega_{k}\left(v_{-}\right) \omega_{k}\left(-v_{\sigma}\right), \\
& =-\omega_{k}\left(v_{-}-\left(v_{\alpha}, y_{0}\right)\right) \omega_{k}\left(-v_{\sigma}+\left(0, y_{0}\right)\right)
\end{aligned}
$$

hence $2 G\left(k_{1}, k_{2}\right)=1+\omega_{k}\left(v_{\sigma}-\left(0, y_{0}\right)\right)+\omega_{k}\left(v_{-}-\left(v_{\alpha}, y_{0}\right)\right)\left(\overline{\omega_{k}\left(v_{\sigma}-\left(0, y_{0}\right)\right)}-1\right)$. For $\omega_{k}\left(v_{\sigma}-\left(0, y_{0}\right)\right)=z_{1}$ and $\omega_{k}\left(v_{-}-\left(v_{\alpha}, y_{0}\right)\right)=z_{2}$, the equation $G\left(k_{1}, k_{2}\right)=0$ is equivalent to $\left(1+z_{1}\right) /\left(1-\overline{z_{1}}\right)=z_{2}$ because $z_{1}=1$ is not a solution of $G\left(k_{1}, k_{2}\right)=0$. Therefore, $\left|\left(1+z_{1}\right) /\left(1-\overline{z_{1}}\right)\right|=1$ which implies $\operatorname{Re}\left(z_{1}\right)=0 \Leftrightarrow \omega_{k}\left(v_{\sigma}-\left(0, y_{0}\right)\right)= \pm i$ and $z_{2}=\omega_{k}\left(v_{-}-\left(v_{\alpha}, y_{0}\right)\right)=1$, leading to $k \in\left(\mathcal{M}_{-}^{*} \cap \mathcal{N}_{\tilde{\sigma}}^{*}\right)$.

Let $\mathcal{P}^{*}$ be the complement of $\mathcal{O}^{*}$ in $\mathcal{L}^{*}$ :

$$
\mathcal{P}^{*}=\left\{k \in \mathcal{L}^{*}: \mathrm{J}^{I d}(k) \neq \mathbf{J}^{I d} \vee \mathrm{J}^{\alpha}(k) \neq \mathbf{J}^{\alpha}\right\} .
$$

In Lemma 7.4 we reformulate the cases of Lemma 7.2 in terms of $\mathcal{L}^{*}$ instead of $\mathcal{O}^{*}$. The first two cases of Lemma 7.2 cannot occur since $\mathcal{P}^{*}$ is too small. In the remaining cases we show that $\mathcal{P}^{*}$ may be ignored and, therefore, that $\mathcal{L}^{*}$ can 
replace $\mathcal{O}^{*}$ in the expressions given. Thus, the estimate of the size of $\mathcal{P}^{*}$ in the next lemma is an essential step.

Lemma 7.3. $\mathcal{P}^{*}$ is the intersection of $\mathcal{L}^{*}$ with the union of a finite number of vector subspaces of $\mathbf{R}^{n+1}$ with codimension at least one.

Proof. $\mathcal{P}^{*}$ is the union of the submodules

$$
\bigcup_{\delta \in \mathbf{J}-\mathbf{J}^{I d}} \mathcal{M}_{\delta, I d}^{*} \cup \bigcup_{\delta \in \mathbf{J}-\mathbf{J}^{\alpha}} \mathcal{M}_{\delta, \alpha}^{*}
$$

where $\mathcal{M}_{\delta, I d}^{*}=\left\{k \in \mathcal{L}^{*}: \delta \in \mathrm{J}^{I d}(k)\right\}$ and $\mathcal{M}_{\delta, \alpha}^{*}=\left\{k \in \mathcal{L}^{*}: \delta \in \mathrm{J}^{\alpha}(k)\right\}$. This union is finite because $\mathbf{J}$ is finite. Moreover, for all $\xi \neq I d_{n+1} \in \mathbf{O}(n+1), \operatorname{Fix}(\xi)=$ $\left\{(x, y) \in \mathbf{R}^{n+1}: \xi(x, y)=(x, y)\right\}$ is a proper vector subspace of $\mathbf{R}^{n+1}$.

Let $\delta \in \mathbf{J}-\mathbf{J}^{I d}$. If $k \in \mathcal{M}_{\delta, I d}^{*}$ then either $\delta k=k$ or $\delta k=\sigma k \Leftrightarrow \sigma \delta k=k$, which implies $\mathcal{M}_{\delta, I d}^{*}=\mathcal{L}^{*} \cap(\operatorname{Fix}(\delta) \cup \operatorname{Fix}(\sigma \delta))$. Moreover, neither $\delta=I d_{n+1}$ nor $\sigma \delta=I d_{n+1}$, by the hypothesis $\delta \in \mathbf{J}-\mathbf{J}^{I d}$. Thus, the codimensions of the subspaces $\operatorname{Fix}(\delta)$ and $\operatorname{Fix}(\sigma \delta)$ are at least one.

Analogously, if $\delta \in \mathbf{J}-\mathbf{J}^{\alpha}$ and $k \in \mathcal{M}_{\delta, \alpha}^{*}$ then either $\delta k=\alpha_{+}^{-1} k \Leftrightarrow \alpha_{+} \delta k=k$ or $\delta k=\alpha_{-}^{-1} k \Leftrightarrow \alpha_{-} \delta k=k$. Therefore, $\mathcal{M}_{\delta, \alpha}^{*}=\mathcal{L}^{*} \cap\left(\operatorname{Fix}\left(\alpha_{+} \delta\right) \cup \operatorname{Fix}\left(\alpha_{-} \delta\right)\right)$, where both $\operatorname{Fix}\left(\alpha_{+} \delta\right)$ and $\operatorname{Fix}\left(\alpha_{-} \delta\right)$ have codimensions at least one due to the hypothesis $\delta \in \mathbf{J}-\mathbf{J}^{\alpha}$.

Lemma 7.4. Suppose that the invariance conditions (a) and (b) of Lemma 7.1 hold for all $k=\left(k_{1}, k_{2}\right) \in \mathcal{L}^{*}$. Then we have one of the following cases:

(A) $\mathbf{J}^{\alpha}=\left\{\alpha_{+}^{-1}\right\}$ and $\mathcal{L}^{*}=\mathcal{N}_{y_{0}}^{*} \cup \mathcal{M}_{+}^{*}$,

(B) $\mathbf{J}^{\alpha}=\left\{\alpha_{-}^{-1}\right\}$ and $\mathcal{L}^{*}=\mathcal{N}_{y_{0}}^{*} \cup \mathcal{M}_{-}^{*}$,

(C) $\mathbf{J}^{\alpha}=\left\{\alpha_{+}^{-1}, \alpha_{-}^{-1}\right\}$, $\mathcal{M}^{*} \subset\left(\mathcal{N}_{y_{0}}^{*} \cup \mathcal{M}_{+}^{*} \cup \mathcal{N}_{\sigma}^{*}\right)$ and $\mathcal{N}^{*} \subset\left(\mathcal{N}_{y_{0}}^{*} \cup\left(\mathcal{M}_{-}^{*} \cap \mathcal{N}_{\tilde{\sigma}}^{*}\right)\right)$.

Proof. At first, we prove the statement:

$$
\text { If }(0, a) \in \mathcal{L} \text { for some } a \neq 0 \text { then } \mathcal{M}_{y_{0}}^{*} \not \subset \mathcal{P}^{*} .
$$

If $(0, a) \in \mathcal{L}$ for some $a \neq 0$ then property (2) of the bases, in Lemma 5.2, ensures that $\mathcal{M}_{y_{0}}^{*}$ has $n$ linearly independent generators, $l_{i}^{*}=\left(a_{i}^{*}, 0\right)$ for $i \in\{1, \ldots, n\}$, where $\left\{a_{1}^{*}, \ldots, a_{n}^{*}\right\}_{\mathbf{R}}=\mathbf{R}^{n}$. If $\mathcal{M}_{y_{0}}^{*} \subset \mathcal{P}^{*}$ then, by Lemma 7.3 , the module $\mathcal{M}_{y_{0}}^{*}$ is a subset of one of the subspaces forming $\mathcal{P}^{*}$. Therefore, there is either an element $\delta \in \mathbf{J}-\mathbf{J}^{I d}$ such that $\delta\left(k_{1}, 0\right)=\left(k_{1}, 0\right)$ for all $\left(k_{1}, 0\right) \in \mathcal{M}_{y_{0}}^{*}$, or some $\delta \in \mathbf{J}-\mathbf{J}^{\alpha}$ such that $\delta\left(k_{1}, 0\right)=\left(\alpha^{-1} k_{1}, 0\right)$ for all $\left(k_{1}, 0\right) \in \mathcal{M}_{y_{0}}^{*}$. By orthogonality of $\delta$ the first case implies either $\delta=I$ or $\delta=\sigma$, which is equivalent to $\delta \in \mathbf{J}^{I d}$. Similarly, in the second case $\delta \in \mathbf{J}^{\alpha}$, by orthogonality of $\delta$ and $\alpha$, and the statement is proved.

For any $k \in \mathcal{L}^{*}, k \neq(0,0)$, let $g \neq(0,0)$ be the smallest element of $\mathcal{L}^{*}$ in the direction of $k$. Thus, there are elements $g_{1}, \ldots, g_{n} \in \mathcal{L}^{*}$ such that $\mathcal{L}^{*}=$ $\left\{g, g_{1}, \ldots, g_{n}\right\}_{\mathbf{Z}}$. Let $\mathcal{M}_{k}^{*}$ be the submodule $\mathcal{M}_{k}^{*}=\left\{g_{1}, g_{2}, \ldots, g_{n}\right\}_{\mathbf{Z}} \subset \mathcal{L}^{*}$ and, given $h \in \mathcal{M}_{k}^{*}$, let $\mathcal{Q}_{k, h}^{*}$ be the set $\mathcal{Q}_{k, h}^{*}=\{k+m h: m \in \mathbf{Z}\}$.

We claim that there is some $h \in \mathcal{M}_{k}^{*}$ such that $\mathcal{Q}_{k, h}^{*} \cap \mathcal{P}^{*}$ is a finite set. Lemma 7.3 asserts that $\mathcal{P}^{*} \subset \bigcup_{i=1}^{m} H_{i}$, where each $H_{i}$ is a subspace of $\mathbf{R}^{n+1}$ of codimension one. Let $p \in \mathbf{N}$ and consider the subset of $k+\mathcal{M}_{k}^{*}$ with $p^{n}$ elements:

$$
W_{p}=\left\{k+m_{1} g_{1}+\cdots+m_{n} g_{n}: m_{i} \in \mathbf{Z}, 1 \leq m_{i} \leq p\right\} .
$$

Each $H_{i}$ has at most $p^{n-1}$ elements in $W_{p}$ and so $W_{p} \cap \bigcup_{i=1}^{m} H_{i}$ has, at most, $m p^{n-1}$ elements. For $p>m$ we have $p^{n}>m p^{n-1}$ and there is some $h \in \mathcal{M}^{*}$ such that $k+h \notin \bigcup_{i=1}^{m} H_{i}$. For this $h$, let $r$ be a line containing $\mathcal{Q}_{k, h}^{*}$. Since for each $i, r \cap H_{i}$ is either $r$ or a finite set, and $r$ contains at least the element $k+h \notin H_{i}$, it follows 
that $\bigcup_{i=1}^{m}\left(r \cap H_{i}\right)$ is a finite set. The claim is proved because $\mathcal{Q}_{k, h}^{*} \cap \mathcal{P}^{*}$ is a subset of $\bigcup_{i=1}^{m}\left(r \cap H_{i}\right)$.

Let $k$ be any element of $\mathcal{L}^{*}-\{(0,0)\}$ and choose some $h \in \mathcal{M}_{k}^{*}$ such that $\mathcal{Q}_{k, h}^{*} \cap \mathcal{P}^{*}$ is a finite set. For simplicity of notation we write $\mathcal{Q}^{*}$ instead of $\mathcal{Q}_{k, h}^{*}$.

Since $\overline{\mathcal{N}_{y_{0}}^{*}}$ is a module, the intersection $\mathcal{Q}^{*} \cap \overline{\mathcal{N}_{y_{0}}^{*}}$ is either the empty set or a set with only a point or an infinite set of equally spaced points with a characteristic period, $\tau_{y_{0}}$. For the set $\mathcal{Q}^{*} \cap \mathcal{N}_{\sigma}^{*}$ there are also the three possible results. Although $\mathcal{N}_{\sigma}^{*}$ is not a module, the smallest difference between two elements of $\mathcal{Q}^{*} \cap \mathcal{N}_{\sigma}^{*}$ defines a period $\tau_{\sigma} \in \mathcal{M}_{\sigma}^{*}$, by the properties of $\mathcal{N}_{\sigma}^{*}$ stated before Lemma 4.1. An analogous construction may be done for the sets $\mathcal{Q}^{*} \cap \mathcal{M}_{+}^{*}, \mathcal{Q}^{*} \cap \mathcal{M}_{-}^{*}$ and $\mathcal{Q}^{*} \cap\left(\mathcal{M}_{-}^{*} \cap \mathcal{N}_{\tilde{\sigma}}^{*}\right)$. Thus, if these sets have more than one element we may define characteristic periods $\tau_{+}, \tau_{-}$and $\tau_{\tilde{\sigma}}$, respectively.

Under the hypothesis of the Lemma, one of the cases (1) to (5) of Lemma 7.2 must happen.

If case (1) happens then $\mathcal{L}^{*}=\mathcal{N}_{y_{0}}^{*} \cup \mathcal{P}^{*}$, which implies $\mathcal{M}_{y_{0}}^{*} \subset \mathcal{P}^{*}$. Moreover, $\mathcal{Q}^{*} \cap \mathcal{N}_{y_{0}}^{*}$ must be an infinite set because $\mathcal{Q}^{*} \cap \mathcal{P}^{*}$ is, by construction, finite. Thus, there exists the period $\tau_{y_{0}}$ implying that $\mathcal{Q}^{*}-\overline{\mathcal{N}}_{y_{0}}^{*}$ is either the empty set or an infinite set. Since $\left(\mathcal{Q}^{*}-\overline{\mathcal{N}_{y_{0}}^{*}}\right) \subset\left(\mathcal{Q}^{*} \cap \mathcal{P}^{*}\right)$ is finite, it follows that $\mathcal{L}^{*}=\overline{\mathcal{N}_{y_{0}}^{*}}$, which implies that $\left(0, y_{0}\right) \in \mathcal{L}$. However, by the statement (12), under this condition, $\mathcal{M}_{y_{0}}^{*}$ cannot be a subset of $\mathcal{P}^{*}$ and so case (1) cannot occur.

In case $(2), \mathcal{L}^{*}=\mathcal{N}_{y_{0}}^{*} \cup \mathcal{N}_{\sigma}^{*} \cup \mathcal{P}^{*}$ which implies $\mathcal{M}_{y_{0}}^{*} \subset\left(\mathcal{N}_{\sigma}^{*} \cup \mathcal{P}^{*}\right)$. Moreover, there is an element $(0, a) \in \mathcal{L}$, with $a \neq 0$, due to the existence of $\sigma$ in $\mathbf{J}$, (see properties (2) and (3) of Lemma 5.2), and thus $\mathcal{M}_{y_{0}}^{*} \cap \mathcal{N}_{\sigma}^{*} \neq \emptyset$. Suppose $\tilde{k} \in \mathcal{M}_{y_{0}}^{*}-\mathcal{P}^{*}$ and $\tilde{k} \neq(0,0)$. Thus, $\tilde{k} \in \mathcal{N}_{\sigma}^{*}$ and $2 \tilde{k} \in \mathcal{M}_{y_{0}}^{*}$. However, by the properties of $\mathcal{N}_{\sigma}^{*}$, $2 \tilde{k} \notin \mathcal{N}_{\sigma}^{*}$ and, by Lemma 7.3, $2 \tilde{k} \notin \mathcal{P}^{*}$. Therefore, case (2) is also impossible.

For case (3) we follow the arguments of case (1). As $\mathcal{L}^{*}=\mathcal{N}_{y_{0}}^{*} \cup \mathcal{M}_{+}^{*} \cup \mathcal{P}^{*}$ then $\mathcal{Q}^{*} \cap\left(\mathcal{N}_{y_{0}}^{*} \cup \mathcal{M}_{+}^{*}\right)$ is an infinite set and at least one of the periods $\tau_{y_{0}}$ or $\tau_{+}$must exist. The least common multiple of the existing periods is a period of $\mathcal{Q}^{*} \cap\left(\mathcal{N}_{y_{0}}^{*} \cup \mathcal{M}_{+}^{*}\right)$ which implies that $\mathcal{Q}^{*}-\left(\mathcal{N}_{y_{0}}^{*} \cup \mathcal{M}_{+}^{*}\right)$ is the empty set. Therefore $k \in\left(\mathcal{N}_{y_{0}}^{*} \cup \mathcal{M}_{+}^{*}\right)$ and condition (A) follows by definition of $k$ and because $(0,0) \in$ $\mathcal{M}_{+}^{*}$.

In a similar way, with $\mathcal{M}_{-}^{*}$ and $\tau_{-}$instead of $\mathcal{M}_{+}^{*}$ and $\tau_{+}$, we prove that case (4) of Lemma 7.2 leads to condition (B).

In case (5) $\left(\mathcal{Q}^{*} \cap \mathcal{M}^{*}\right)-\left(\mathcal{N}_{y_{0}}^{*} \cup \mathcal{M}_{+}^{*} \cup \mathcal{N}_{\sigma}^{*}\right)$ must be the empty set by the necessary existence of, at least, one of the periods $\tau_{y_{0}}, \tau_{+}$or $\tau_{\sigma}$ and, analogously, $\left(\mathcal{Q}^{*} \cap \mathcal{N}^{*}\right)-\left(\mathcal{N}_{y_{0}}^{*} \cup\left(\mathcal{M}_{-}^{*} \cap \mathcal{N}_{\tilde{\sigma}}^{*}\right)\right)$ is empty due to the least common multiple of the periods $\tau_{y_{0}}$ and $\tau_{\tilde{\sigma}}$. Besides, either $k \in\left(\mathcal{Q}^{*} \cap \mathcal{M}^{*}\right)$ or $k \in\left(\mathcal{Q}^{*} \cap \mathcal{N}^{*}\right)$ and, as $(0,0) \notin \mathcal{N}^{*}$, condition $(\mathrm{C})$ follows.

This completes the proof of Proposition 4.1.

\section{RESTRICTION}

In this section we present results for the restriction of functions in $X_{\Gamma}$ analogous to those obtained for the projection.

Recall that for $r \in \mathbf{R}$, the operator $\Phi_{r}$ maps $f(x, y)$ to its restriction to the affine subspace $\left\{(x, r): x \in \mathbf{R}^{n}\right\}$ given by $\Phi_{r}(f)(x)=f(x, r)$. If $f \in X_{\Gamma}$ then, the restriction of $f$ is

$$
\Phi_{r}(f)(x)=\sum_{k \in \mathcal{L}^{*}} \omega_{k}(x, r) C(k)=\sum_{k_{1} \in \mathcal{L}_{1}^{*}} \omega_{k_{1}}(x) D\left(k_{1}\right)
$$

where $\mathcal{L}_{1}^{*}=\left\{k_{1}:\left(k_{1}, k_{2}\right) \in \mathcal{L}^{*}\right\}$ and $D\left(k_{1}\right)=\sum_{k_{2}:\left(k_{1}, k_{2}\right) \in \mathcal{L}^{*}} C\left(k_{1}, k_{2}\right) \omega_{k_{2}}(r)$. 
Theorem 8.1. All functions in $\Phi_{r}\left(X_{\Gamma}\right)$ are invariant under the action of $\left(v_{\alpha}, \alpha\right) \in$ $\mathbf{R}^{n} \ltimes \mathbf{O}(n)$ if and only if one of the following conditions holds:

(I) $\left(\left(v_{\alpha}, 0\right), \alpha_{+}\right) \in \Gamma$,

(II) $\left(\left(v_{\alpha}, 2 r\right), \alpha_{-}\right) \in \Gamma$.

Given $f \in X_{\Gamma}$, the Fourier series for $\Phi_{r}(f)$ is similar to that of $\Pi_{y_{0}}(f)$, with $\omega_{k_{2}}(r)$ in the restriction corresponding to $\int_{0}^{y_{0}} \omega_{k_{2}}(y) d y$ in the projection. Thus, results concerning $\Phi_{r}$ are similar to those proved in the previous sections for $\Pi_{y_{0}}$. In particular, the proof of Theorem 8.1 is analogous to that of Theorem 1.2. The condition $\omega_{k_{2}}(r)=0$ is never verified and so the sets $\mathcal{N}_{y_{0}}^{*}$ and $\mathcal{M}_{y_{0}}^{*}$ disappear and we don't have an analogue to the condition $\left(0, y_{0}\right) \in \mathcal{L}$. Moreover, the expression

$$
\int_{0}^{y_{0}} \omega_{k_{2}}(y) d y-\omega_{k_{2}}\left(y_{0}\right) \int_{0}^{y_{0}} \omega_{-k_{2}}(y) d y=0
$$

has the analogue $\omega_{k_{2}}(r)-\omega_{k_{2}}(2 r) \omega_{-k_{2}}(r)=0$.

The following analogue of Proposition 4.1 is used to prove Theorem 8.1.

Proposition 8.1. All functions in $\Phi_{r}\left(X_{\Gamma}\right)$ are invariant under the action of $\left(v_{\alpha}, \alpha\right) \in$ $\mathbf{R}^{n} \ltimes \mathbf{O}(n)$ if and only if one of the following conditions holds:

(A) $\left(v_{+}, \alpha_{+}\right) \in \Gamma$ and $\mathcal{L}^{*}=\mathcal{M}_{+}^{*}$,

(B) $\left(v_{-}, \alpha_{-}\right) \in \Gamma$ and $\mathcal{L}^{*}=\mathcal{M}_{-}^{*}$,

(C) both $\left(v_{\sigma}, \sigma\right)$ and $\left(v_{+}, \alpha_{+}\right)$belong to $\Gamma, \mathcal{M}^{*} \subset\left(\mathcal{M}_{+}^{*} \cup \mathcal{N}_{\sigma}^{*}\right)$ and $\mathcal{N}^{*} \subset$ $\left(\mathcal{M}_{-}^{*} \cap \mathcal{N}_{\tilde{\sigma}}^{*}\right)$.

The analogue of Lemma 7.2 is, for $D^{\prime}(\delta, k)=\omega_{\delta k}\left(-v_{\delta}\right) \omega_{\left.\delta k\right|_{2}}(r)$ :

Lemma 8.1. Suppose that

(a) if $k_{1} \in \mathcal{L}_{1}^{*} \cap \alpha \mathcal{L}_{1}^{*}$ then $\sum_{\delta \in \mathrm{J}^{I d}(k)} D^{\prime}(\delta, k)=\omega_{k_{1}}\left(-v_{\alpha}\right) \sum_{\delta \in \mathrm{J}^{\alpha}(k)} D^{\prime}(\delta, k)$ and

(b) if $k_{1} \notin \mathcal{L}_{1}^{*} \cap \alpha \mathcal{L}_{1}^{*}$ then $\sum_{\delta \in \mathrm{J}^{I d}(k)} D^{\prime}(\delta, k)=0$,

for all $k=\left(k_{1}, k_{2}\right) \in \mathcal{L}^{*}$. Then one of the following cases holds:

(1) $\mathbf{J}^{I d}=\left\{I d_{n+1}, \sigma\right\}, \mathbf{J}^{\alpha}=\emptyset$ and $\mathcal{O}^{*} \subset \mathcal{N}_{\sigma}^{*}$,

(2) $\mathbf{J}^{I d}=\left\{I d_{n+1}\right\}, \mathbf{J}^{\alpha}=\left\{\alpha_{+}^{-1}\right\}$ and $\mathcal{O}^{*} \subset \mathcal{M}_{+}^{*}$,

(3) $\mathbf{J}^{I d}=\left\{I d_{n+1}\right\}, \mathbf{J}^{\alpha}=\left\{\alpha_{-}^{-1}\right\}$ and $\mathcal{O}^{*} \subset \mathcal{M}_{-}^{*}$,

(4) $\mathbf{J}^{I d}=\left\{I d_{n+1}, \sigma\right\}, \mathbf{J}^{\alpha}=\left\{\alpha_{+}^{-1}, \alpha_{-}^{-1}\right\},\left(\mathcal{O}^{*} \cap \mathcal{M}^{*}\right) \subset\left(\mathcal{M}_{+}^{*} \cup \mathcal{N}_{\sigma}^{*}\right)$ and $\left(\mathcal{O}^{*} \cap \mathcal{N}^{*}\right) \subset$ $\left(\mathcal{M}_{-}^{*} \cap \mathcal{N}_{\tilde{\sigma}}^{*}\right)$.

The proof of Proposition 8.1 also uses the analogue of Lemma 7.4. Under the conditions for the restriction, property (12) in the proof of Lemma 7.4, concerning the set $\mathcal{M}_{y_{0}}^{*}$, does not hold. By Lemma 8.1 the case (1) of Lemma 7.4 disappears. For case (2) of Lemma 7.4 the dual lattice is $\mathcal{L}^{*}=\mathcal{N}_{\sigma}^{*} \cup \mathcal{P}^{*}$ and the arguments concerning $\mathcal{M}_{y_{0}}^{*}$ and $\mathcal{N}_{y_{0}}^{*}$ must be replaced by: if $\tilde{k} \notin \mathcal{P}^{*}$ then $\tilde{k} \in \mathcal{N}_{\sigma}^{*}$. However both $2 \tilde{k} \notin \mathcal{P}^{*}$ and $2 \tilde{k} \notin \mathcal{N}_{\sigma}^{*}$, by definition of $\mathcal{P}^{*}$ and the properties of $\mathcal{N}_{\sigma}^{*}$, and so this case is not possible.

\section{EXAMPLES}

We conclude the article with some simple examples to illustrate the dependence on $\Gamma$ of the group $\Sigma_{y_{0}}$ of symmetries of the projected functions $\Pi_{y_{0}}\left(X_{\Gamma}\right)$ and of the group $G_{r}$ of symmetries of the restriction $\Phi_{r}\left(X_{\Gamma}\right)$ of functions in $X_{\Gamma}$ to a hyperplane. We use the notation $\left[\gamma_{1}, \ldots, \gamma_{k}\right]$ for the subgroup of the Euclidean group generated by $\gamma_{1}, \ldots, \gamma_{k}$.

Example 1. Let $A, B \in \mathbf{R}^{2}$ be $A=(\sqrt{3} / 2,1 / 2), B=(0,1)$, generators of a hexagonal lattice $\mathcal{L}^{0}$ in the plane. Take $\Gamma$ to have $\mathcal{L}^{0}$ as its translation subgroup, 
with trivial point group $\mathbf{J}=\left\{I_{2}\right\}$, hence $\widehat{\Gamma}=\Gamma$. If $y_{0} \notin \mathbf{Z}$, then $\left(0, y_{0}\right) \notin \mathcal{L}^{0}$. Conditions (I) of Theorems 1.2 and 8.1 imply that

$$
\Sigma_{y_{0}}=G_{r}=\left[\left(\sqrt{3}, I d_{1}\right)\right] \quad \forall y_{0}>0, y_{0} \notin \mathbf{N} \quad \text { and } \quad \forall r \in \mathbf{R}
$$

obtained from $\left(2 A-B, I d_{2}\right) \in \Gamma$. Applying to $\left(A, I d_{2}\right) \in \Gamma$ the condition (III) of Theorem 1.2 we obtain

$$
\Sigma_{y_{0}}=\left[\left(\sqrt{3} / 2, I d_{1}\right)\right] \quad \forall y_{0} \in \mathbf{N} .
$$

Thus, the projection and restriction of $\Gamma$-invariant functions are always periodic, but for $y_{0} \in \mathbf{N}$, projected functions have half the usual period. Restricted functions do not have the smaller period.

The lattice $\mathcal{L}^{0}$ is an integral lattice: the square of the norm of all its generators is an integer. Tilting the lattice slightly keeps this property and produces a dramatic change in $\Sigma_{y_{0}}$, and $G_{r}$, as shown in the next example.

Example 2. Let $R_{\theta}: \mathbf{R}^{2} \longrightarrow \mathbf{R}^{2}$ be the counterclockwise rotation of an angle $\theta$ around the origin with $\sqrt{3} \tan \theta \notin \mathbf{Q}$, and let $\mathcal{L}^{\theta}=R_{\theta}\left(\mathcal{L}^{0}\right)$. The restriction $\sqrt{3} \tan \theta \notin \mathbf{Q}$ ensures that $\mathcal{L}^{\theta}$ contains no element of the form $(v, 0)$ with $v \neq 0$, nor an element of the form $\left(0, y_{0}\right)$, with $y_{0}>0$. For $\Gamma=\mathcal{L}^{\theta} \ltimes\left\{I d_{2}\right\}$ we have $\Sigma_{y_{0}}=G_{r}=\left\{\left(0, I d_{1}\right)\right\}$ for all $y_{0}>0$ and all $r \in \mathbf{R}$. The projection and restriction of $\Gamma$-invariant functions are not periodic.

Since $\mathcal{L}^{\theta} \cap \mathbf{R} \times\{0\}=\{(0,0)\}$ and $\mathcal{L}^{\theta}$ is an integral lattice, then for small $\theta>0$, the projection $P: \mathbf{R}^{2} \longrightarrow \mathbf{R}^{2}, P(x, y)=x$ maps

$$
\mathcal{L}^{\theta} \cap \mathbf{R} \times[-h, h] \quad \text { with } \quad h=\frac{\sqrt{3}}{6} \sin \theta+\frac{1}{2} \cos \theta
$$

into a quasi-periodic set (see Senechal [17, Ch. 2]). This is the canonical projection that was mentioned at the end of section 1.1.

The holohedry of the lattice $\mathcal{L}^{0}$ of example 1 is the group $\mathbf{D}_{6}$ generated by the reflection on the horizontal axis $\sigma$ and by the rotation of $\pi / 3$ around the origin.

Example 3. Here $\Gamma$ is given by $\Gamma=\mathcal{L}^{\theta} \ltimes \mathbf{D}_{6}$ (called p6mm in Armstrong [1, Ch.26]) where $\mathbf{D}_{6}$ is generated by the rotation of $\pi / 3$ around the origin and the reflection on the line through the origin that makes an angle $\theta$ with the horizontal axis. Then $\widehat{\Gamma}=\mathcal{L}^{\theta} \ltimes\left\{ \pm I d_{2}\right\}$ and for most values of $y_{0}$ and $r$ we still get $\Sigma_{y_{0}}=G_{r}=$ $\left\{\left(0, I d_{1}\right)\right\}$, from condition (I) of Theorem 1.2 and condition (I) of Theorem 8.1. For some special values of $y_{0}, \Sigma_{y_{0}}$ contains a reflection $\left(a,-I d_{1}\right)$ for some $a \neq 0$ from condition (II) of Theorem 1.2 applied to $\left(l,-I d_{2}\right), l \in \mathcal{L}^{\theta}$. Similarly, $G_{r}$ contains a reflection for some special values of $r$, by condition (II) of Theorem 8.1.

The next example uses a small perturbation of the lattice $\mathcal{L}^{0}$. It is no longer an integral lattice, and has a smaller holohedry. It illustrates the way small modifications in the lattice entail different symmetries in the projection.

Example 4. Let $\Gamma=\mathcal{L}^{\alpha} \ltimes\left\{ \pm I d_{2}\right\}$ where $\mathcal{L}^{\alpha}=\{B, C\}_{\mathbf{Z}}$ for $B=(0,1)$ and $C=(\sqrt{3} / 2, \alpha+1 / 2)$. Note that $\left\{ \pm I d_{2}\right\}$ is the holohedry of $\mathcal{L}^{\alpha}$. If $0<|\alpha|<1 / 2$ then $\mathcal{L}^{\alpha}$ is not an integral lattice, and if $\alpha \notin \mathbf{Q}$ then $\mathcal{L}^{\alpha} \cap\{(x, 0) x \in \mathbf{R}\}=\{(0,0)\}$. In this case:

- if $y_{0} \in \mathbf{N}$, then $\Sigma_{y_{0}}=\left[\left(0,-I d_{1}\right),\left(\sqrt{3} / 2, I d_{1}\right)\right]$ by condition (III) of Theorem 1.2;

- if $y_{0}=m(\alpha+1 / 2)+n, m, n \in \mathbf{N}$, then $\Sigma_{y_{0}}=\left[\left(m \sqrt{3} / 2,-I d_{1}\right)\right]$ by condition (II) of Theorem 1.2;

- if $2 r=m(\alpha+1 / 2)+n, m, n \in \mathbf{Z}$, then $G_{r}=\left[\left(m \sqrt{3} / 2,-I d_{1}\right)\right]$ by condition (II) of Theorem 8.1; 
- for all other values of $y_{0}$ and of $r$, we get $\Sigma_{y_{0}}=\left\{\left(0, I d_{1}\right)\right\}=G_{r}$.

Therefore, for most choices of $y_{0}$ and of $r$, the projection and the restriction of all functions in $X_{\Gamma}$ do not have any symmetries, but projection from a band of integer thickness produces periodic functions, in contrast to example 3.

Crystallographic groups acting on $\mathbf{R}^{3}$ have a richer structure, as the next examples show.

Example 5. Let $\mathcal{L}^{f}$ be the face centered cubic lattice (fcc) with generators $D=$ $(0,1,0), E=(\sqrt{3} / 2,1 / 2,0)$ and $F=(\sqrt{3} / 6,1 / 2, \sqrt{6} / 3)$. The vertical elements of $\mathcal{L}^{f}$ are of the form $(0,0, n \sqrt{6})$, with $n \in \mathbf{Z}$. Take $\Gamma$ to have $\mathcal{L}^{f}$ as its translation subgroup, with trivial point group $\mathbf{J}=\left\{I_{3}\right\}$, hence $\widehat{\Gamma}=\Gamma$ and

- for all values of $r$, we have $G_{r}=\left[\left((\sqrt{3} / 2,1 / 2), I d_{2}\right),\left((0,1), I d_{2}\right)\right]$;

- if $y_{0} \neq n \sqrt{6}$, with $n \in \mathbf{N}$, then $\Sigma_{y_{0}}=\left[\left((\sqrt{3} / 2,1 / 2), I d_{2}\right),\left((0,1), I d_{2}\right)\right]$;

- if $y_{0}=n \sqrt{6}$, with $n \in \mathbf{N}$, then $\left.\left.\Sigma_{y_{0}}=\left[\left((\sqrt{3} / 6,1 / 2), I d_{2}\right)\right),\left((0,1), I d_{2}\right)\right)\right]$ by condition (III) of Theorem 1.2.

The symmetry group of the restriction of $\Gamma$-invariant functions is that of example 1 , with lattice $\mathcal{L}^{0}$, coinciding with the symmetries of most projections. However, for some values of $y_{0}$, the projections of all $\Gamma$-invariant functions have smaller periods.

The holohedry of the lattice $\mathcal{L}^{f}$ in example 5 is the group of symmetries of the cube, that does not contain any rotation of order 6 . Hence, if we take $\Gamma$ with translation subgroup $\mathcal{L}^{f}$ and with the rotational symmetries of the cube as its point group, then, for most values of $y_{0}$, the translation subgroup of $\Sigma_{y_{0}}$ is $\mathcal{L}^{0}$ but with point group that is not its full holohedry.

Example 6. Consider a group $\Gamma=\mathcal{L}^{f} \ltimes \mathbf{J}$ with the lattice $\mathcal{L}^{f}$ of example 5 and point group $\mathbf{J}$ generated by $\alpha_{-}$and $\beta_{-}$where $\alpha$ and $\beta$ are the elements of $\mathbf{O}(2)$ :

$$
\alpha=\left(\begin{array}{cc}
1 / 2 & -\sqrt{3} / 2 \\
\sqrt{3} / 2 & 1 / 2
\end{array}\right) \quad \text { and } \beta=\left(\begin{array}{cc}
-1 & 0 \\
0 & 1
\end{array}\right) \text {. }
$$

In this case $\widehat{\Gamma}=\Gamma$ and we claim that, using the terminology of Armstrong [1, Ch.26]:

- if $y_{0} \neq n \sqrt{6} / 3$, with $n \in \mathbf{N}$, then $\Sigma_{y_{0}}$ is the group p31m;

- if $r \neq n \sqrt{6} / 6$, with $n \in \mathbf{Z}$, then $G_{r}$ is the group p31m;

- if $y_{0}=n \sqrt{6} / 3$, with $n \in \mathbf{N}$, then $\Sigma_{y_{0}}$ is the group p6mm;

- if $r=n \sqrt{6} / 6$, with $n \in \mathbf{Z}$, then $G_{r}$ is the group p6mm.

To establish the claims in example 6 , note that if $y_{0} \neq n \sqrt{6} / 3$, with $n \in \mathbf{N}$, then the only applicable condition in Theorem 1.2 is condition (I), since there are no elements in $\Gamma$ with translation part of the form $\left(a, b, y_{0}\right), a, b \in \mathbf{R}$. The lattice of $\Sigma_{y_{0}}$ is $\mathcal{L}^{0}$, as in example 5 . The point group of $\Gamma_{y_{0}}$ contains the $2 \pi / 3$ rotations around the vertical axis, $\left(\alpha_{-}\right)^{2}$ and $\left(\alpha_{-}\right)^{4}$, and the reflections on vertical planes $\left(\beta_{-}\right)\left(\alpha_{-}\right),\left(\beta_{-}\right)\left(\alpha_{-}\right)^{3},\left(\beta_{-}\right)\left(\alpha_{-}\right)^{5}$. The point group of $\Sigma_{y_{0}}$ is $\left[\alpha^{2}, \beta \alpha\right]$. All these orthogonal maps occur in $\Sigma_{y_{0}}$ with translation part $(0,0)$. The same applies to $\widetilde{\Gamma}_{2 r}$ if $r \neq n \sqrt{6} / 6$, with $n \in \mathbf{Z}$.

For $y_{0}=n \sqrt{6}$, with $n \in \mathbf{N}$, all the conditions of Theorem 1.2 are applicable and the group $\Sigma_{y_{0}}$ is p6mm, with lattice generated by $(\sqrt{3} / 6,1 / 2)$ and $(0,1)$, as in example 5 , and point group generated by $\alpha$ and $\beta$, with $((0,0), \alpha)$ and $((0,0), \beta) \in \Gamma$.

If $y_{0}=n \sqrt{6} / 3$, with $n \in \mathbf{N}, n \not \equiv 0(\bmod 3)$ then $\left(0,0, y_{0}\right) \notin \mathcal{L}^{f}$, so condition (III) of Theorem 1.2 is not applicable, but condition (II) is. Then $(n(\sqrt{3} / 6,1 / 2), \alpha) \in$ $\Sigma_{y_{0}}$ by condition (II) applied to $\left(n F, \alpha_{-}\right) \in \Gamma$. Similarly, $(n(\sqrt{3} / 6,1 / 2), \beta) \in \Sigma_{y_{0}}$. Since the translation subgroup of $\Sigma_{y_{0}}$ is the hexagonal lattice $\mathcal{L}^{0}$ and since $\Sigma_{y_{0}}$ contains a rotation of order 6 , then it is p6mm. The group $G_{r}$ is the same for 
$r=n \sqrt{6} / 6$, with $n \in \mathbf{Z}$. Note that this is a different representation of $\mathrm{p} 6 \mathrm{~mm}$, here the translation subgroup is $\mathcal{L}^{0}$ and the rotation of order 6 does not fix the origin.

\section{ACKNOWLEDGEMENTS}

We would like to thank Marjorie Senechal for many helpful comments and suggestions.

The research of the two authors at Centro de Matemática da Universidade do Porto (CMUP) was funded by the European Regional Development Funding FEDER through the programme COMPETE and by the Portuguese Government through the FCT - Fundação para a Ciência e a Tecnologia under the project PEst-C/MAT/UI0144/2011. E.M. Pinho was partly supported by the grant SFRH/BD/13334/2003 of FCT and by UBI- Universidade da Beira Interior, Portugal.

\section{REFERENCES}

[1] M. A. Armstrong, Groups and Symmetry, Springer-Verlag, 1988

[2] P. Borckmans, G. Dewel, A. De Wit, E. Dulos, J. Boissonade, F. Gauffre and P. De Kepper, 'Diffusive instabilities and chemical reactions', Int. J. Bifurcation and Chaos 12 (2002) 23072332

[3] T. K. Callahan, 'Hopf bifurcation on cubic lattices', in J. Buescu, S. B. S. D. Castro, A. P. S. Dias and I. S. Labouriau (Eds.) Bifurcations, Symmetry and Patterns Birkhäuser, Series: Trends in Mathematics (2003) pages 123-127

[4] T. K. Callahan and E. Knobloch, 'Symmetry-breaking bifurcations on cubic lattices', Nonlinearity 10 (1997) 1179-1216

[5] T. K. Callahan and E. Knobloch, 'Pattern formation in three-dimensional reaction-diffusion systems', Physica D 132 (1999) 339-362

[6] P. De Kepper, E. Dulos, J. Boissonade, A. De Wit, G. Dewel and P. Borckmans, 'Reactiondiffusion patterns in confined chemical systems', J. Stat. Phys. 101 (2000) 495-508

[7] B. Dionne and M. Golubitsky, 'Planforms in two and three dimensions', Z. Angew. Math. Phys. 43 (1992) 36-62

[8] E. Dulos, P. Davies, B. Rudovics and P. De Kepper, 'From quasi-2D to 3D Turing patterns in ramped systems', Physica D 98 (1996) 53-66

[9] M. Golubitsky and I. Stewart, The Symmetry Perspective, Birkhäuser-Verlag, 2002

[10] M. G. M. Gomes, 'Black-eye patterns: A representation of three-dimensional symmetries in thin domains', Phys. Rev. E 60 (1999) 3741-3747

[11] G.H. Gunaratne, Q. Ouyang, H.L. Swinney, 'Pattern formation in the presence of symmetries', Phys. Rev. E 50 (1994) 2802-2820

[12] I. S. Labouriau and E. M. Pinho, 'Symmetries of projected wallpaper patterns', Math. Proc. Camb. Phil. Soc. 141 (2006) 421-441

[13] I. Melbourne, 'Steady-state bifurcation with Euclidean symmetry', Trans. Amer. Math. Soc. 351 (1999) 1575-1603

[14] W. Miller, Symmetry Groups and their Applications, Academic Press, 1972

[15] M. J. Parker, I. Stewart and M. G. M. Gomes, 'Examples of forced symmetry-breaking to homoclinic cycles in three-dimensional euclidean-invariant systems', Int. J. Bif. Chaos 18 (2008) 83-107

[16] E. M. Pinho, Symmetries of Projected Symmetric Patterns, PhD Thesis, University of Porto, Portugal, 2006

[17] M. Senechal, Quasicrystals and Geometry, Cambridge University Press, 1995

[18] H. Shoji, K. Yamada, D. Ueyama and T. Ohta, 'Turing patterns in three dimensions', Phys. Rev. E 75 (2007) 046212

[19] G.P. Tolstov, Fourier Series, Dover, 1976

[20] C. Zhou, H. Guo and Q. Ouyang, 'Experimental study of the dimensionality of black-eye patterns', Phys. Rev. E 65 (2002) 036118

E. M. Pinho - Departamento de Matemática, Faculdade de Ciências da Universidade do Porto, Rua do Campo Alegre, 687, 4169-007 Porto, Portugal

E-mail address: empinho@gmail.com

Isabel S. Labouriau - Centro de Matemática da Universidade do Porto, Rua do Campo Alegre, 687, 4169-007 Porto, Portugal

E-mail address: islabour@fc.up.pt 